\title{
An extension of matroid rank submodularity and the $Z$-Rayleigh property
}

\author{
Arun P. Mani \\ Clayton School of Information Technology \\ Monash University, Clayton VIC 3800, Australia \\ arunpmani@gmail.com
}

Submitted: Mar 24, 2011; Accepted: May 6, 2011; Published: May 16, 2011

Mathematics Subject Classification: 05B35

\begin{abstract}
We define an extension of matroid rank submodularity called $R$-submodularity, and introduce a minor-closed class of matroids called extended submodular matroids that are well-behaved with respect to $R$-submodularity. We apply $R$-submodularity to study a class of matroids with negatively correlated multivariate Tutte polynomials called the $Z$-Rayleigh matroids. First, we show that the class of extended submodular matroids are $Z$-Rayleigh. Second, we characterize a minor-minimal non- $Z$-Rayleigh matroid using its $R$-submodular properties. Lastly, we use $R$ submodularity to show that the Fano and non-Fano matroids (neither of which is extended submodular) are $Z$-Rayleigh, thus giving the first known examples of $Z$-Rayleigh matroids without the half-plane property.
\end{abstract}

\section{Introduction and background}

One of the fundamental axioms of a matroid rank function is its submodular property. For a matroid, $M(E, \rho)$, this is stated as follows [11, p.23].

(SM) For all $X, Y \subseteq E, \rho(X \cup Y)+\rho(X \cap Y) \leq \rho(X)+\rho(Y)$.

In this paper we define the following extension of submodularity. For mutually disjoint $P_{1}, P_{2}, R \subseteq E$, we say $P_{1}$ and $P_{2}$ are $R$-submodular in $M$ if there exists a bijection $\pi: 2^{R} \rightarrow 2^{R}$ such that for all $C \subseteq R, \rho\left(P_{1} \cup P_{2} \cup C\right)+\rho(R \backslash C) \leq \rho\left(P_{1} \cup \pi C\right)+\rho\left(P_{2} \cup R \backslash \pi C\right)$. Under our definition, the property (SM) is equivalent to the $\emptyset$-submodularity of sets $X \backslash Y$ and $Y \backslash X$ in the minor $M / X \cap Y$ for all $X, Y \subseteq E$. We further say the matroid $M(E, \rho)$ is extended submodular if for all mutually disjoint subsets $P_{1}, P_{2}, R \subseteq E$, the sets $P_{1}$ and $P_{2}$ are $R$-submodular in $M$ and its minors. We show that the class of all extended 
submodular matroids is closed under some fundamental matroid operations, and includes the uniform matroids and series-parallel networks among others.

A primary application of extended submodularity is in the study of Rayleigh properties of the multivariate Tutte polynomial of matroids. Following Sokal [13], we define the multivariate Tutte polynomial of a matroid $M(E, \rho)$ to be

$$
Z(M, q ; \mathbf{y})=\sum_{A \subseteq E} q^{-\rho(A)} \prod_{e \in A} y_{e}
$$

where $q$ and $\mathbf{y}=\left(y_{e}: e \in E\right)$ are commuting indeterminates. (An equivalent function called the Tugger polynomial was defined earlier by Kung [7].) We refer to [13] for the many useful properties of $Z(M, q ; \mathbf{y})$. The Tutte polynomial of $M(E, \rho)$ is the two variable polynomial

$$
T(M ; x, y)=\sum_{A \subseteq E}(x-1)^{\rho(E)-\rho(A)}(y-1)^{|A|-\rho(A)},
$$

and is known to be a special case of $Z(M, q ; \mathbf{y})$. For $e, f \in E$ let

$$
\begin{aligned}
\Delta Z\{e, f\} & (M, q ; \mathbf{y})=Z(M / e \backslash f, q ; \mathbf{y}) \cdot Z(M \backslash e / f, q ; \mathbf{y}) \\
& -q^{\rho(\{e\})+\rho(\{f\})-\rho(\{e, f\})} Z(M / e / f, q ; \mathbf{y}) \cdot Z(M \backslash e \backslash f, q ; \mathbf{y}) .
\end{aligned}
$$

Sokal [14] calls $M(E, \rho) Z$-Rayleigh if for all distinct $e, f \in E, 0<q \leq 1$ and $\mathbf{y}>0$, $\Delta Z\{e, f\}(M, q ; \mathbf{y}) \geq 0$. Here we use the notation $\mathbf{y}>0$ to denote $y_{e}>0$ for all $e \in E$. We shall often consider $\Delta Z\{e, f\}(M, q ; \mathbf{y})$ to be a multivariate polynomial, called a $Z$-Rayleigh difference polynomial, in the elements of $\mathbf{y}$. Note that our definition of $\Delta Z\{e, f\}(M, q ; \mathbf{y})$ follows Wagner [16, Section 3].

The generating polynomial of the bases of a matroid $M(E, \rho)$ is

$$
B(M ; \mathbf{y})=\sum_{\substack{A \subseteq E \\ A \in \mathcal{B}}} \prod_{e \in A} y_{e}
$$

where $\mathcal{B}$ is the collection of bases of $M$ [7]. Choe and Wagner [4] initiated the discussion on Rayleigh matroids by calling $M(E, \rho)$ Rayleigh if for all distinct $e, f \in E$ and $\mathbf{y}>0$, $\Delta B\{e, f\}(M ; \mathbf{y})=B(M / e \backslash f ; \mathbf{y}) B(M \backslash e / f ; \mathbf{y})-B(M / e / f ; \mathbf{y}) B(M \backslash e \backslash f ; \mathbf{y}) \geq 0$. Matroids with analogous properties for the collection of independent and spanning sets were termed independence correlated and spanning correlated matroids by Semple and Welsh [12] and Cocks [5]. In the rest of this paper, we follow Wagner [16] and use the terms B-Rayleigh, I-Rayleigh and S-Rayleigh for Rayleigh, independence correlated and spanning correlated matroids, respectively. Wagner [16] also called $M(E, \rho)$ PottsRayleigh if $\Delta Z\{e, f\}(M, q ; \mathbf{y}) \geq 0$ for all distinct $e, f \in E$ with $q$ in some interval $0<q \leq$ $q_{*}(M) \leq 1$ and $\mathbf{y}>0$. It is easy to show that all $Z$-Rayleigh matroids are Potts-Rayleigh, and all Potts-Rayleigh matroids are also $B$-Rayleigh, $I$-Rayleigh and $S$-Rayleigh. Semple and Welsh [12] further showed that the class B-Rayleigh includes both the $I$-Rayleigh and $S$-Rayleigh matroids. However, no other inclusion relationship is known among these classes. 
It is known that all uniform matroids and series parallel networks are $Z$-Rayleigh [16]. In this paper, we show that every extended submodular matroid is $Z$-Rayleigh with the additional property that all coefficients of its $Z$-Rayleigh difference polynomials are nonnegative for all $q$ in the interval $0<q \leq 1$. This provides a combinatorial explanation for the occurrences of only non-negative coefficients in the $B$-Rayleigh, $I$-Rayleigh and $S$ Rayleigh difference polynomials of some matroids as reported in [12], [5] and [16]. More generally, for any $e, f \in E$, we show that the $R$-submodularity of sets $\{e\}$ and $\{f\}$ in a minor of $M(E, \rho)$ is a sufficient condition for non-negativity of a corresponding coefficient in $\Delta Z\{e, f\}(M, q ; \mathbf{y})$ whenever $0<q \leq 1$. Even when a matroid is not extended submodular, this result allows us to quickly test the non-negativity of the coefficients of its $Z$-Rayleigh difference polynomials for all $q$ in $0<q \leq 1$, which in turn reduces the amount of computation required to verify if the matroid is $Z$-Rayleigh. We illustrate this by showing that the Fano and non-Fano matroids are $Z$-Rayleigh.

The remainder of the paper is organized as follows. The next section defines $R$ submodularity in matroids, and Section 3 introduces the class of extended submodular matroids. Section 4 discusses the relationship between $R$-submodularity and the $Z$-Rayleigh property of matroids. We conclude with a discussion of open problems in Section 5 . Throughout the paper, we assume familiarity with fundamental matroid concepts and notations [11]. Additionally, if $N$ is a minor of matroid $M(E, \rho)$, we use $\rho_{N}$ to denote the rank function of $N$. Finally, we note that the term extended submodular inequality has been used previously with a meaning very different from ours by Greene and Magnanti [6, Section 3] for an inequality that is valid in all matroids, and by Bouchet [1, Section 2] as an axiom for multimatroids.

\section{$2 \quad R$-submodularity in matroids}

The notion of $R$-submodularity is based on matroid rank dominations that were introduced in $[9]$.

Given three mutually disjoint sets, $P_{1}, P_{2}, R$, we first define a set, $S\left(P_{1}, P_{2}, R\right)$, of disjoint pairs as follows.

$$
S\left(P_{1}, P_{2}, R\right)=\left\{\left(P_{1} \cup C, P_{2} \cup R \backslash C\right): C \subseteq R\right\} .
$$

Equivalently, the set $S\left(P_{1}, P_{2}, R\right)$ is the collection of all partitions $(X, Y)$ of the set $P_{1} \cup$ $P_{2} \cup R$ subject to the constraints $P_{1} \subseteq X$ and $P_{2} \subseteq Y$. There is one such pair $(X, Y)$ for every subset of $R$, and hence $\left|S\left(P_{1}, P_{2}, R\right)\right|=2^{|R|}$.

For the sake of brevity, henceforth any reference to the set $S\left(P_{1}, P_{2}, R\right)$ will be understood to imply that the three sets $P_{1}, P_{2}$ and $R$ are mutually disjoint.

Definition 1 (Rank domination and equivalence [9]). Let $M(E, \rho)$ be a matroid and $P_{1}, P_{2}, Q_{1}, Q_{2}, R \subseteq E$ such that $\left(P_{1}, P_{2}, R\right)$ and $\left(Q_{1}, Q_{2}, R\right)$ are triples of mutually disjoint sets. We say the set $S\left(P_{1}, P_{2}, R\right)$ is rank dominated by $S\left(Q_{1}, Q_{2}, R\right)$ in $M$ if there exists a bijection $\pi: 2^{R} \rightarrow 2^{R}$ such that for all $C \subseteq R, \rho\left(P_{1} \cup C\right)+\rho\left(P_{2} \cup R \backslash C\right) \leq$ $\rho\left(Q_{1} \cup \pi C\right)+\rho\left(Q_{2} \cup R \backslash \pi C\right)$. We write $S\left(P_{1}, P_{2}, R\right) \leq_{M} S\left(Q_{1}, Q_{2}, R\right)$ to denote such a 
relationship, omitting the subscript $M$ when the matroid is clear from the context, and call $\pi$ a rank dominating bijection of the 4-tuple $\left(P_{1}, P_{2}, Q_{1}, Q_{2}\right)$ in $M$.

Additionally, if for all $C \subseteq R, \rho\left(P_{1} \cup C\right)+\rho\left(P_{2} \cup R \backslash C\right)=\rho\left(Q_{1} \cup \pi C\right)+\rho\left(Q_{2} \cup R \backslash\right.$ $\pi C)$, we also say the set $S\left(P_{1}, P_{2}, R\right)$ is rank equivalent to $S\left(Q_{1}, Q_{2}, R\right)$ in $M$, and write $S\left(P_{1}, P_{2}, R\right) \equiv_{M} S\left(Q_{1}, Q_{2}, R\right)$ (omitting the subscript $M$ if the matroid is clear from the context).

Note that despite a notational change in the definition of a rank dominating bijection from the one used in [9, Section 2], it is easy to check that the two definitions are equivalent. The following properties of rank domination and equivalence were shown in [9].

Proposition 2 (Mani [9]). In any matroid $M(E, \rho)$ for all $P_{1}, P_{2}, Q_{1}, Q_{2}, T_{1}, T_{2}, R \subseteq E$ :

1. (Reflexivity). $S\left(P_{1}, P_{2}, R\right) \equiv S\left(P_{1}, P_{2}, R\right)$. (Thus rank domination is also reflexive.)

2. (Transitivity). If $S\left(P_{1}, P_{2}, R\right) \leq S\left(Q_{1}, Q_{2}, R\right)$ and $S\left(Q_{1}, Q_{2}, R\right) \leq S\left(T_{1}, T_{2}, R\right)$, then $S\left(P_{1}, P_{2}, R\right) \leq S\left(T_{1}, T_{2}, R\right)$. (Hence rank equivalence is also transitive.)

3. (Symmetry of $\equiv)$. If $S\left(P_{1}, P_{2}, R\right) \equiv S\left(Q_{1}, Q_{2}, R\right)$ then $S\left(Q_{1}, Q_{2}, R\right) \equiv S\left(P_{1}, P_{2}, R\right)$.

4. (Antisymmetry of $\leq)$. $S\left(P_{1}, P_{2}, R\right) \leq S\left(Q_{1}, Q_{2}, R\right)$ and $S\left(Q_{1}, Q_{2}, R\right) \leq S\left(P_{1}, P_{2}, R\right)$ if and only if $S\left(P_{1}, P_{2}, R\right) \equiv S\left(Q_{1}, Q_{2}, R\right)$.

5. $S\left(P_{1}, P_{2}, R\right) \equiv S\left(P_{2}, P_{1}, R\right)$.

Rank submodularity provides an useful example of rank dominations as shown in our next example.

Example 3. In any matroid $M(E, \rho)$, for all disjoint pairs $P_{1}, P_{2} \subseteq E$, we have $S\left(P_{1} \cup\right.$ $\left.P_{2}, \emptyset, \emptyset\right) \leq S\left(P_{1}, P_{2}, \emptyset\right)$. This can be shown to be equivalent to the property ( $\mathbf{S M}$ ).

\subsection{Definition and properties}

We now define $R$-submodularity as follows.

Definition 4 (R-submodularity). Let $M(E, \rho)$ be a matroid and $R \subseteq E$. We say two disjoint sets $P_{1}, P_{2} \subseteq E \backslash R$ are $R$-submodular in $M$ if there exists a bijection $\pi: 2^{R} \rightarrow 2^{R}$ such that for all $C \subseteq R, \rho\left(P_{1} \cup P_{2} \cup C\right)+\rho(R \backslash C) \leq \rho\left(P_{1} \cup \pi C\right)+\rho\left(P_{2} \cup R \backslash \pi C\right)$. We call $\pi$ an $R$-submodular bijection of the ordered pair $\left(P_{1}, P_{2}\right)$ in $M$.

Additionally, if for all $C \subseteq R, \rho\left(P_{1} \cup P_{2} \cup C\right)+\rho(R \backslash C)=\rho\left(P_{1} \cup \pi C\right)+\rho\left(P_{2} \cup R \backslash \pi C\right)$, we say $P_{1}$ and $P_{2}$ are $R$-modular in $M$, and $\pi$ an $R$-modular bijection of the ordered pair $\left(P_{1}, P_{2}\right)$.

Equivalently, $P_{1}$ and $P_{2}$ are $R$-submodular in $M$ if $S\left(P_{1} \cup P_{2}, \emptyset, R\right) \leq_{M} S\left(P_{1}, P_{2}, R\right)$, and $R$-modular if $S\left(P_{1} \cup P_{2}, \emptyset, R\right) \equiv_{M} S\left(P_{1}, P_{2}, R\right)$. The next result is easy to establish for all $M(E, \rho)$.

Proposition 5. 1. All disjoint $P_{1}, P_{2} \subseteq E$ are $\emptyset$-submodular in $M$. (See Example 3.) 


\section{For all disjoint $P, R \subseteq E$, the sets $P$ and $\emptyset$ are $R$-modular in $M$.}

We further know of the following instances of $R$-submodularity for a matroid $M(E, \rho)$. The first of these was communicated to us by Noble [10]. Recall that the closure operator in $M$ is the map $\mathrm{cl}_{M}: 2^{E} \rightarrow 2^{E}$ defined by $\operatorname{cl}_{M}(X)=\{e: \rho(X \cup\{e\})=\rho(X)\}$.

Proposition 6 (Noble [10]). All disjoint $P_{1}, P_{2} \subseteq E$ are $R$-submodular in $M$ whenever $R \subseteq \operatorname{cl}_{M}\left(P_{1} \cup P_{2}\right) \backslash\left(P_{1} \cup P_{2}\right)$.

Proposition 7 (Mani [9]). All disjoint $P_{1}, P_{2} \subseteq E$ are $R$-submodular in $M$ whenever $R \subseteq E \backslash\left(P_{1} \cup P_{2}\right)$ and $|R| \leq 3$.

However, as the following counterexamples demonstrate, in general Proposition 7 is false if $|R|>3$.

Example 8. Consider the matroids $W_{3}$ and $\mathcal{W}_{3}$ with geometric representations as shown in Figure 1. $W_{3}$ and $\mathcal{W}_{3}$ are called the rank-three wheel and whirl matroids, respectively [11, p.293]. If we let $P_{1}=\{1\}, P_{2}=\{4\}$ and $R=\{2,3,5,6\}$, then Table 1 shows that $P_{1}$ and $P_{2}$ are not $R$-submodular in $W_{3}$ and $\mathcal{W}_{3}$.
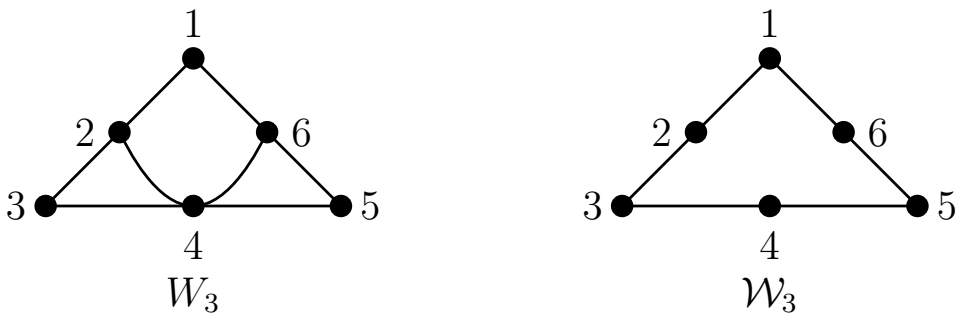

Figure 1: Rank 3-wheel and whirl

Nevertheless, in Section 3 we introduce a minor closed class of matroids where all disjoint $P_{1}, P_{2} \subseteq E$ are $R$-submodular whenever $R \subseteq E \backslash\left(P_{1} \cup P_{2}\right)$.

We next look at the effect of some common matroid operations on its $R$-submodularity. First, the deletion operation is easily seen to preserve $R$-submodularity.

Proposition 9. Let $M(E, \rho)$ be a matroid. Then for all mutually disjoint $P_{1}, P_{2}, R \subseteq E$ and $F \subseteq E \backslash\left(P_{1} \cup P_{2} \cup R\right), \pi: 2^{R} \rightarrow 2^{R}$ is an $R$-submodular bijection of the pair $\left(P_{1}, P_{2}\right)$ in $M$ if and only if $\pi$ is an $R$-submodular bijection of $\left(P_{1}, P_{2}\right)$ in $M \backslash F$.

Proof. Use $\rho_{M \backslash F}(X)=\rho(X)$ for all $X \subseteq E \backslash F$ in Definition 4.

In contrast, the next counterexample demonstrates that a contraction operation (and by inference, also duality) does not necessarily preserve $R$-submodularity. 


\begin{tabular}{|c|c|c|c|c|}
\hline \multirow{2}{*}{$C$} & \multicolumn{2}{|c|}{$W_{3}$} & \multicolumn{2}{|c|}{$\mathcal{W}_{3}$} \\
\cline { 2 - 5 } & $\rho(\{1,4\} \cup C)+$ & $\rho(\{1\} \cup C)+$ & $\rho(\{1,4\} \cup C)+$ & $\rho(\{1\} \cup C)+$ \\
& $\rho(\{2,3,5,6\} \backslash C)$ & $\rho(\{2,3,4,5,6\} \backslash C)$ & $\rho(\{2,3,5,6\} \backslash C)$ & $\rho(\{2,3,4,5,6\} \backslash C)$ \\
\hline$\emptyset$ & 5 & 4 & 5 & 4 \\
$\{2\}$ & 6 & 5 & 6 & 5 \\
$\{3\}$ & 6 & 5 & 6 & 5 \\
$\{5\}$ & 6 & 5 & 6 & 5 \\
$\{6\}$ & 6 & 5 & 6 & 5 \\
$\{2,3\}$ & 5 & 5 & 5 & 5 \\
$\{2,5\}$ & 5 & 6 & 5 & 6 \\
$\{2,6\}$ & 5 & 5 & 5 & 5 \\
$\{3,5\}$ & 5 & 5 & 5 & 6 \\
$\{3,6\}$ & 5 & 6 & 5 & 5 \\
$\{5,6\}$ & 5 & 5 & 5 & 5 \\
$\{2,3,5\}$ & 4 & 5 & 4 & 5 \\
$\{2,3,6\}$ & 4 & 5 & 4 & 5 \\
$\{2,5,6\}$ & 4 & 5 & 4 & 5 \\
$\{3,5,6\}$ & 4 & 5 & 4 & 4 \\
$\{2,3,5,6\}$ & 3 & 4 & 3 & \\
\hline
\end{tabular}

Table 1: $\{1\}$ and $\{4\}$ are not $\{2,3,5,6\}$-submodular in $W_{3}$ and $\mathcal{W}_{3}$. (Submodular bijections in these two cases are impossible as columns two and four contain more number of 6's than columns three and five, respectively).

Example 10. Let $M(G)$ be the cycle matroid of graph $G$ with edges labeled as shown in Figure 2. Also let $P_{1}=\{1\}, P_{2}=\{4\}$ and $R=\{2,3,5,6\}$. Then it can be checked from Proposition 5-2 and Lemma 14-1 below, that $P_{1}$ and $P_{2}$ are $R$-submodular in $M(G)$, while Example 8 implies that $P_{1}$ and $P_{2}$ are not $R$-submodular in $M(G) / 7=W_{3}$.

Despite this observation, our next result shows that an $R$-submodularity relationship in $M(E, \rho)$ manifests itself in a particular minor of its dual matroid. The dual of $M(E, \rho)$ is the matroid $M^{*}\left(E, \rho^{*}\right)$, where for all $X \subseteq E$,

$$
\rho^{*}(X)=|X|-\rho(E)+\rho(E \backslash X) .
$$

Proposition 11. Let $M(E, \rho)$ be a matroid. Then for all mutually disjoint $P_{1}, P_{2}, R \subseteq E$, $\pi: 2^{R} \rightarrow 2^{R}$ is an $R$-submodular bijection of the pair $\left(P_{1}, P_{2}\right)$ in $M$ if and only if $\pi$ is an $R$-submodular bijection of $\left(P_{1}, P_{2}\right)$ in $M^{*} / F$, where $F=E \backslash\left(P_{1} \cup P_{2} \cup R\right)$.

Proof. By definition, $\pi: 2^{R} \rightarrow 2^{R}$ is an $R$-submodular bijection of $\left(P_{1}, P_{2}\right)$ in $M$ if and only if for all $C \subseteq R$,

$$
\rho\left(P_{1} \cup P_{2} \cup C\right)+\rho(R \backslash C) \leq \rho\left(P_{1} \cup \pi C\right)+\rho\left(P_{2} \cup R \backslash \pi C\right) .
$$




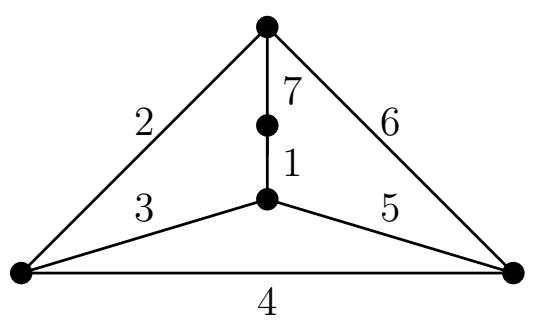

Figure 2: A Graph $G$ such that $M(G) / 7=W_{3}$

Since $F \cup P_{1} \cup P_{2} \cup R=E$, we also have

$$
\begin{gathered}
P_{1} \cup P_{2} \cup C=E \backslash(F \cup R \backslash C), \quad R \backslash C=E \backslash\left(F \cup P_{1} \cup P_{2} \cup C\right) \\
P_{1} \cup \pi C=E \backslash\left(F \cup P_{2} \cup R \backslash \pi C\right), \text { and } P_{2} \cup R \backslash \pi C=E \backslash\left(F \cup P_{1} \cup \pi C\right) .
\end{gathered}
$$

It follows that $\pi$ is an $R$-submodular bijection of $\left(P_{1}, P_{2}\right)$ in $M$ if and only if for all $C \subseteq R$, $\rho(E \backslash(F \cup R \backslash C))+\rho\left(E \backslash\left(F \cup P_{1} \cup P_{2} \cup C\right)\right) \leq \rho\left(E \backslash\left(F \cup P_{2} \cup R \backslash \pi C\right)\right)+\rho\left(E \backslash\left(F \cup P_{1} \cup \pi C\right)\right)$.

Now, applying (3) we see, equivalently, for all $C \subseteq R$,

$$
\rho^{*}\left(F \cup P_{1} \cup P_{2} \cup C\right)+\rho^{*}(F \cup R \backslash C) \leq \rho^{*}\left(F \cup P_{1} \cup \pi C\right)+\rho^{*}\left(F \cup P_{2} \cup R \backslash \pi C\right) .
$$

That is, $\pi$ is an $R$-submodular bijection of $\left(P_{1}, P_{2}\right)$ in both $M$ and $M^{*} / F$.

When $P_{1} \cup P_{2} \cup R=E$, we get the following useful corollary.

Corollary 12. Let $M(E, \rho)$ be a matroid, and $P_{1}, P_{2}, R \subseteq E$ be three mutually disjoint sets such that $P_{1} \cup P_{2} \cup R=E$. Then $\pi: 2^{R} \rightarrow 2^{R}$ is an $R$-submodular bijection of $\left(P_{1}, P_{2}\right)$ in $M$ if and only if $\pi$ is an $R$-submodular bijection of $\left(P_{1}, P_{2}\right)$ in $M^{*}$.

If $M_{1}\left(E_{1}, \rho_{1}\right)$ and $M_{2}\left(E_{2}, \rho_{2}\right)$ are two matroids defined on disjoint sets $E_{1}$ and $E_{2}$, then their direct sum is the matroid $M_{1} \oplus M_{2}(E, \rho)$, where $E=E_{1} \cup E_{2}$, and $\rho(X)=$ $\rho_{1}\left(X \cap E_{1}\right)+\rho_{2}\left(X \cap E_{2}\right)$ for all $X \subseteq E$. We now show that $R$-submodularity extends itself over the direct sum of matroids.

Proposition 13. Let $M_{1}\left(E_{1}, \rho_{1}\right)$ and $M_{2}\left(E_{2}, \rho_{2}\right)$ be two matroids such that $E_{1}$ and $E_{2}$ are disjoint. Also let $P_{11}, P_{21}, R_{1} \subseteq E_{1}$ and $P_{21}, P_{22}, R_{2} \subseteq E_{2}$ such that $P_{11}$ and $P_{21}$ are $R_{1}$-submodular in $M_{1}$, and $P_{12}$ and $P_{22}$ are $R_{2}$-submodular in $M_{2}$. Then $P_{11} \cup P_{12}$ and $P_{21} \cup P_{22}$ are $R_{1} \cup R_{2}$-submodular in the matroid $M_{1} \oplus M_{2}$.

Proof. For $i=1,2$, let $\pi_{i}: 2^{R_{i}} \rightarrow 2^{R_{i}}$ be an $R_{i}$-submodular bijection of the pair $\left(P_{1 i}, P_{2 i}\right)$ in $M_{i}$. Also let $P_{1}=P_{11} \cup P_{12}, P_{2}=P_{21} \cup P_{22}$ and $R=R_{1} \cup R_{2}$. It is straightforward to verify that the bijection $\pi: 2^{R} \rightarrow 2^{R}$ defined by, for all $C \subseteq R, \pi C=\pi_{1}\left(C \cap R_{1}\right) \cup \pi_{2}\left(C \cap R_{2}\right)$ is an $R$-submodular bijection of $\left(P_{1}, P_{2}\right)$ in $M_{1} \oplus M_{2}$. 


\subsection{Useful $R$-submodularity constructions}

Our next series of results shows $R$-submodularity in minors can sometimes be used to construct larger pairs of $R$-submodular sets in a matroid.

Lemma 14. Let $M(E, \rho)$ be a matroid, $P_{1}, P_{2}, R \subseteq E$ and $p \in E$ such that $P_{1}$ and $P_{2}$ are $R$-submodular in $M \backslash p$.

1. If $p \in \operatorname{cl}_{M}\left(P_{1} \cup P_{2}\right)$, then for $i, j \in\{1,2\}, i \neq j$, sets $P_{i} \cup\{p\}$ and $P_{j}$ are $R$-submodular in $M$.

2. For $i, j \in\{1,2\}, i \neq j$, if $p \in E \backslash \mathrm{cl}_{M}\left(P_{i} \cup R\right)$ then:

(a) $P_{i} \cup\{p\}$ and $P_{j}$ are $R$-submodular in $M$, and

(b) $S\left(P_{i} \cup P_{j},\{p\}, R\right) \leq_{M} S\left(P_{i} \cup\{p\}, P_{j}, R\right)$.

Proof. Let $\pi: 2^{R} \rightarrow 2^{R}$ be an $R$-submodular bijection of $\left(P_{1}, P_{2}\right)$ in $M \backslash p$. Then, from Proposition $9, \pi$ is also an $R$-submodular bijection of $\left(P_{1}, P_{2}\right)$ in $M$. Hence for all $C \subseteq R$,

$$
\rho\left(P_{1} \cup P_{2} \cup C\right)+\rho(R \backslash C) \leq \rho\left(P_{1} \cup \pi C\right)+\rho\left(P_{2} \cup R \backslash \pi C\right) .
$$

(1). We prove the case $i=1, j=2$ below, and skip the similar proof when $i=2, j=1$.

When $p \in \mathrm{cl}_{M}\left(P_{1} \cup P_{2}\right)$, we know for all $C \subseteq R, \rho\left(P_{1} \cup P_{2} \cup\{p\} \cup C\right)=\rho\left(P_{1} \cup P_{2} \cup C\right)$. Since $\rho(X) \leq \rho(X \cup\{p\})$ for all $X \subseteq E$ (4) implies, for all $C \subseteq R$,

$$
\rho\left(P_{1} \cup P_{2} \cup\{p\} \cup C\right)+\rho(R \backslash C) \leq \rho\left(P_{1} \cup\{p\} \cup \pi C\right)+\rho\left(P_{2} \cup R \backslash \pi C\right) .
$$

In other words, $\pi$ is also an $R$-submodular bijection of the pair $\left(P_{1} \cup\{p\}, P_{2}\right)$.

(2). We prove the case $i=1, j=2$, and omit the similar proof for the case $i=2, j=1$.

When $p \in E \backslash \operatorname{cl}_{M}\left(P_{1} \cup R\right)$, we know for all $C \subseteq R, p \notin \operatorname{cl}_{M}\left(P_{1} \cup \pi C\right)$ and so $\rho\left(P_{1} \cup\{p\} \cup \pi C\right)=\rho\left(P_{1} \cup \pi C\right)+1$. Also $\rho(X \cup\{p\}) \leq \rho(X)+1$ for all $X \subseteq E$, and thus (4) implies for all $C \subseteq R$,

$$
\rho\left(P_{1} \cup P_{2} \cup\{p\} \cup C\right)+\rho(R \backslash C) \leq \rho\left(P_{1} \cup\{p\} \cup \pi C\right)+\rho\left(P_{2} \cup R \backslash \pi C\right),
$$

and

$$
\rho\left(P_{1} \cup P_{2} \cup C\right)+\rho(\{p\} \cup R \backslash C) \leq \rho\left(P_{1} \cup\{p\} \cup \pi C\right)+\rho\left(P_{2} \cup R \backslash \pi C\right) .
$$

Thus $\pi$ is an $R$-submodular bijection of the pair $\left(P_{1} \cup\{p\}, P_{2}\right)$, and a rank dominating bijection of the 4-tuple $\left(P_{1} \cup P_{2},\{p\}, P_{1} \cup\{p\}, P_{2}\right)$ in $M$.

Lemma 15. Let $M(E, \rho)$ be a matroid, $P_{1}, P_{2}, R \subseteq E$ and $p \in E$ such that $P_{1}$ and $P_{2}$ are $R$-submodular in $M / p$.

1. If $p \in E \backslash \mathrm{cl}_{M}(R)$, then for $i, j \in\{1,2\}, i \neq j$, sets $P_{i} \cup\{p\}$ and $P_{j}$ are $R$-submodular in $M$.

2. For $i, j \in\{1,2\}, i \neq j$, if $p \in \operatorname{cl}_{M}\left(P_{i}\right)$ then: 
(a) $P_{i}$ and $P_{j} \cup\{p\}$ are $R$-submodular in $M$, and

(b) $S\left(P_{i} \cup P_{j},\{p\}, R\right) \leq_{M} S\left(P_{i}, P_{j} \cup\{p\}, R\right)$.

Proof. Let $\pi: 2^{R} \rightarrow 2^{R}$ be an $R$-submodular bijection of $\left(P_{1}, P_{2}\right)$ in $M / p$. Then, for all $C \subseteq R$

$$
\rho_{M / p}\left(P_{1} \cup P_{2} \cup C\right)+\rho_{M / p}(R \backslash C) \leq \rho_{M / p}\left(P_{1} \cup \pi C\right)+\rho_{M / p}\left(P_{2} \cup R \backslash \pi C\right) .
$$

Since $\rho_{M / p}(X)=\rho(X \cup\{p\})-\rho(\{p\})$ for all $X \subseteq E \backslash\{p\}$, this can be rewritten as, for all $C \subseteq R$,

$$
\rho\left(P_{1} \cup P_{2} \cup\{p\} \cup C\right)+\rho(\{p\} \cup R \backslash C) \leq \rho\left(P_{1} \cup\{p\} \cup \pi C\right)+\rho\left(P_{2} \cup\{p\} \cup R \backslash \pi C\right) .
$$

(1). We prove the case $i=1, j=2$. The proof for $i=2, j=1$ is similar.

When $p \in E \backslash \mathrm{cl}_{M}(R)$, for all $C \subseteq R, \rho(\{p\} \cup(R \backslash C))=\rho(R \backslash C)+1$. Using $\rho(X) \geq \rho(X \cup\{p\})-1$ for all $X \subseteq E$, we get from (5),

$$
\rho\left(P_{1} \cup P_{2} \cup\{p\} \cup C\right)+\rho(R \backslash C) \leq \rho\left(P_{1} \cup\{p\} \cup \pi C\right)+\rho\left(P_{2} \cup R \backslash \pi C\right),
$$

for all $C \subseteq R$. Hence $\pi$ is an $R$-submodular bijection of the pairs $\left(P_{1} \cup\{p\}, P_{2}\right)$ and $\left(P_{1}, P_{2} \cup\{p\}\right)$ in $M$.

(2). Again we only prove the case $i=1, j=2$.

When $p \in \mathrm{cl}_{M}\left(P_{1}\right)$, for all $C \subseteq R, \rho\left(P_{1} \cup\{p\} \cup \pi C\right)=\rho\left(P_{1} \cup \pi C\right)$. Now applying $\rho(X) \leq \rho(X \cup\{p\})$ for all $X \subseteq E$ to (5) we obtain

$$
\rho\left(P_{1} \cup P_{2} \cup\{p\} \cup C\right)+\rho(R \backslash C) \leq \rho\left(P_{1} \cup \pi C\right)+\rho\left(P_{2} \cup\{p\} \cup R \backslash \pi C\right),
$$

and

$$
\rho\left(P_{1} \cup P_{2} \cup C\right)+\rho(\{p\} \cup R \backslash C) \leq \rho\left(P_{1} \cup \pi C\right)+\rho\left(P_{2} \cup\{p\} \cup R \backslash \pi C\right),
$$

for all $C \subseteq R$. Thus $\pi$ is an $R$-submodular bijection of $\left(P_{1}, P_{2} \cup\{p\}\right)$ and a rank dominating bijection of the 4-tuple $\left(P_{1} \cup P_{2},\{p\}, P_{1}, P_{2} \cup\{p\}\right)$ in $M$.

Lemma 16. Let $M(E, \rho)$ be a matroid, $P_{1}, P_{2}, R \subseteq E$ and $r \in E$ such that $P_{1}$ and $P_{2}$ are $R$-submodular in minors $M \backslash r$ and $M / r$. If $r \in \mathrm{cl}_{M}\left(P_{i}\right)$ or $r \in E \backslash \mathrm{cl}_{M}\left(P_{i} \cup R\right)$ for some $i \in\{1,2\}$, then $P_{1}$ and $P_{2}$ are $R \cup\{r\}$-submodular in $M$.

Proof. Note that,

$$
\begin{aligned}
S\left(P_{1} \cup P_{2}, \emptyset, R \cup\{r\}\right) & =S\left(P_{1} \cup P_{2} \cup\{r\}, \emptyset, R\right) \cup S\left(P_{1} \cup P_{2},\{r\}, R\right), \\
\text { and } S\left(P_{1}, P_{2}, R \cup\{r\}\right) & =S\left(P_{1} \cup\{r\}, P_{2}, R\right) \cup S\left(P_{1}, P_{2} \cup\{r\}, R\right),
\end{aligned}
$$

where all unions are over disjoint sets. We look at two possible cases.

Case $r \in \operatorname{cl}_{M}\left(P_{i}\right), i \in\{1,2\}:$ We prove the case $r \in \operatorname{cl}_{M}\left(P_{1}\right)$, and omit the similar proof of the case $r \in \mathrm{cl}_{M}\left(P_{2}\right)$. 
When $r \in \operatorname{cl}_{M}\left(P_{1}\right)$ we know from Lemma 14-1 that $S\left(P_{1} \cup P_{2} \cup\{r\}, \emptyset, R\right) \leq_{M} S\left(P_{1} \cup\right.$ $\left.\{r\}, P_{2}, R\right)$, and from Lemma 15-2(b) that $S\left(P_{1} \cup P_{2},\{r\}, R\right) \leq_{M} S\left(P_{1}, P_{2} \cup\{r\}, R\right)$. Hence from (6a)-(6b), $S\left(P_{1} \cup P_{2}, \emptyset, R \cup\{r\}\right) \leq_{M} S\left(P_{1}, P_{2}, R \cup\{r\}\right)$.

Case $r \in E \backslash \operatorname{cl}_{M}\left(P_{i} \cup R\right), i \in\{1,2\}$ : We prove the case $r \in E \backslash \operatorname{cl}_{M}\left(P_{1} \cup R\right)$, and the proof when $r \in E \backslash \mathrm{cl}_{M}\left(P_{2} \cup R\right)$ is similar.

When $r \in E \backslash \operatorname{cl}_{M}\left(P_{1} \cup R\right)$, we have from Lemma 14-2(b), $S\left(P_{1} \cup P_{2},\{r\}, R\right) \leq_{M}$ $S\left(P_{1} \cup\{r\}, P_{2}, R\right)$, and from Lemma 15-1, $S\left(P_{1} \cup P_{2} \cup\{r\}, \emptyset, R\right) \leq_{M} S\left(P_{1}, P_{2} \cup\{r\}, R\right)$. The result then follows from (6a)-(6b).

Lemma 17. Let $M(E, \rho)$ be a matroid, $P_{1}, P_{2}, R \subseteq E$ and $r \in E$ such that $P_{1}$ and $P_{2}$ be $R$-submodular in $M \backslash r$ and $M / r$. If there exists an element $s \in R$ such that $\{r, s\}$ is a circuit or a cocircuit in $M$, then $P_{1}$ and $P_{2}$ are $R \cup\{r\}$-submodular in $M$.

Proof. We consider the two cases separately.

Case $1(\{r, s\}$ is a circuit in $M)$ : Let $R^{\prime}=R \backslash\{s\}$. Also let,

$$
\begin{aligned}
& S_{1}=S\left(P_{1} \cup P_{2} \cup\{r, s\}, \emptyset, R^{\prime}\right) \cup S\left(P_{1} \cup P_{2},\{r, s\}, R^{\prime}\right), \\
& S_{2}=S\left(P_{1} \cup P_{2} \cup\{r\},\{s\}, R^{\prime}\right) \cup S\left(P_{1} \cup P_{2} \cup\{s\},\{r\}, R^{\prime}\right), \\
& S_{3}=S\left(P_{1} \cup\{r, s\}, P_{2}, R^{\prime}\right) \cup S\left(P_{1}, P_{2} \cup\{r, s\}, R^{\prime}\right), \text { and } \\
& S_{4}=S\left(P_{1} \cup\{r\}, P_{2} \cup\{s\}, R^{\prime}\right) \cup S\left(P_{1} \cup\{s\}, P_{2} \cup\{r\}, R^{\prime}\right),
\end{aligned}
$$

where all unions are over disjoint sets. Clearly, then

$$
\begin{gathered}
S\left(P_{1} \cup P_{2}, \emptyset, R \cup\{r\}\right)=S_{1} \cup S_{2}, \text { and } \\
S\left(P_{1}, P_{2}, R \cup\{r\}\right)=S_{3} \cup S_{4} .
\end{gathered}
$$

For $i, j \in\{1,2,3,4\}$ we say $S_{i} \leq_{M} S_{j}$ if there exists a bijection $\sigma: S_{i} \rightarrow S_{j}$ such that $\rho(W)+\rho(Z) \leq \rho(X)+\rho(Y)$ whenever $\sigma(W, Z)=(X, Y)$. To prove $S\left(P_{1} \cup P_{2}, \emptyset, R \cup\right.$ $\{r\}) \leq_{M} S\left(P_{1}, P_{2}, R \cup\{r\}\right)$, note that it is enough to show that $S_{1} \leq_{M} S_{3}$ and $S_{2} \leq_{M} S_{4}$.

Since $\{r, s\}$ is a circuit in $M$, using the rank dominating bijection defined by $\pi C=C$ for all $C \subseteq R^{\prime}$, we have

$$
\begin{gathered}
S\left(P_{1} \cup P_{2} \cup\{r, s\}, \emptyset, R^{\prime}\right) \equiv_{M} S\left(P_{1} \cup P_{2} \cup\{s\}, \emptyset, R^{\prime}\right), \\
S\left(P_{1} \cup P_{2},\{r, s\}, R^{\prime}\right) \equiv_{M} S\left(P_{1} \cup P_{2},\{s\}, R^{\prime}\right), \\
S\left(P_{1} \cup\{r, s\}, P_{2}, R^{\prime}\right) \equiv_{M} S\left(P_{1} \cup\{s\}, P_{2}, R^{\prime}\right), \text { and } \\
S\left(P_{1}, P_{2} \cup\{r, s\}, R^{\prime}\right) \equiv_{M} S\left(P_{1}, P_{2} \cup\{s\}, R^{\prime}\right) .
\end{gathered}
$$

However, by definition,

$$
\begin{aligned}
S\left(P_{1} \cup P_{2}, \emptyset, R\right) & =S\left(P_{1} \cup P_{2} \cup\{s\}, \emptyset, R^{\prime}\right) \cup S\left(P_{1} \cup P_{2},\{s\}, R^{\prime}\right), \text { and } \\
S\left(P_{1}, P_{2}, R\right) & =S\left(P_{1} \cup\{s\}, P_{2}, R^{\prime}\right) \cup S\left(P_{1}, P_{2} \cup\{s\}, R^{\prime}\right) .
\end{aligned}
$$

Since $P_{1}$ and $P_{2}$ are $R$-submodular in $M \backslash r$, it follows that,

$S\left(P_{1} \cup P_{2} \cup\{s\}, \emptyset, R^{\prime}\right) \cup S\left(P_{1} \cup P_{2},\{s\}, R^{\prime}\right) \leq_{M} S\left(P_{1} \cup\{s\}, P_{2}, R^{\prime}\right) \cup S\left(P_{1}, P_{2} \cup\{s\}, R^{\prime}\right)$. 
From (7a), (7c) and (8a)-(8d), this is equivalent to $S_{1} \leq_{M} S_{3}$.

Also when $\{r, s\}$ is a circuit in $M, s$ is a loop in $M / r$. Thus, if $P_{1}$ and $P_{2}$ are $R$ submodular in $M / r$, then they are also $R^{\prime}$-submodular in $M / r$, and so

$$
S\left(P_{1} \cup P_{2}, \emptyset, R^{\prime}\right) \leq_{M / r} S\left(P_{1}, P_{2}, R^{\prime}\right)
$$

Using the facts $\rho_{M / r}(X)=\rho(X \cup\{r\})-\rho(\{r\})$ for all $X \subseteq E \backslash\{r\}$, and $\{r, s\}$ is a circuit in $M$ in (9), we obtain

$$
\begin{aligned}
& S\left(P_{1} \cup P_{2} \cup\{r\},\{s\}, R^{\prime}\right) \equiv_{M} S\left(P_{1} \cup P_{2} \cup\{s\},\{r\}, R^{\prime}\right) \leq_{M} \\
& S\left(P_{1} \cup\{s\}, P_{2} \cup\{r\}, R^{\prime}\right) \equiv_{M} S\left(P_{1} \cup\{r\}, P_{2} \cup\{s\}, R^{\prime}\right) .
\end{aligned}
$$

From (7b), (7d) and (10), it follows that $S_{3} \leq_{M} S_{4}$, which proves this case.

Case $2(\{r, s\}$ is a cocircuit in $M)$ : Let $F=E \backslash\left(P_{1} \cup P_{2} \cup R\right)$, and consider the matroid $N=M^{*} / F$. Since $P_{1}$ and $P_{2}$ are $R$-submodular in $M \backslash r$ and $M / r$, from Proposition 11, they are also $R$-submodular in $N \backslash r$ and $N / r$.

Further, the set $\{r, s\}$ is a cocircuit in $M \backslash F$, and hence is a circuit in $N=M^{*} / F$. Thus, using the previous case, $P_{1}$ and $P_{2}$ are $R \cup\{r\}$-submodular in $N$. Finally, another application of Proposition 11 gives $P_{1}$ and $P_{2}$ are $R \cup\{r\}$-submodular in $M$.

\section{$3 \quad$ Extended submodular matroids}

Considering Example 8, we may ask if there exist minor closed classes of matroids in which $P_{1}$ and $P_{2}$ are $R$-submodular for all mutually disjoint $P_{1}, P_{2}, R \subseteq E$. In this section we show that there are some well-known classes of matroids with this property.

\subsection{Definition and properties}

Definition 18 ( $R$-family of minors). Let $M(E, \rho)$ be a matroid. Given an $R \subseteq E$, we define the $R$-family of minors of $M, \mathcal{M F}(M, R)$, to be

$$
\mathcal{M F}(M, R)=\{M / C \backslash(R \backslash C): C \subseteq R\}
$$

That is, the $R$-family of minors is the set of all minors of $M$ obtained by deleting or contracting every element in $R$.

Definition 19 (Extended submodular matroids). A matroid $M(E, \rho)$ is extended submodular if for all mutually disjoint $P_{1}, P_{2}, R \subseteq E$ and minors $N \in \mathcal{M F}\left(M, E \backslash\left(P_{1} \cup\right.\right.$ $\left.\left.P_{2} \cup R\right)\right), P_{1}$ and $P_{2}$ are $R$-submodular in N. We denote the class of extended submodular matroids by $\mathcal{E} \mathcal{S} \mathcal{M}$.

We first show that the class $\mathcal{E} \mathcal{S M}$ is closed under some well-known matroid operations.

Proposition 20. If $M(E, \rho) \in \mathcal{E S M}$ then $M^{*} \in \mathcal{E S M}$. 
Proof. Let $P_{1}, P_{2}, R \subseteq E$, and $N^{*} \in \mathcal{M F}\left(M^{*}, E \backslash\left(P_{1} \cup P_{2} \cup R\right)\right)$. Clearly, if $N=\left(N^{*}\right)^{*}$, then $N \in \mathcal{M F}\left(M, E \backslash\left(P_{1} \cup P_{2} \cup R\right)\right)$. By definition, we know $P_{1}$ and $P_{2}$ are $R$-submodular in $N$. Since the ground set of $N$ is $P_{1} \cup P_{2} \cup R$, from Corollary 12, sets $P_{1}$ and $P_{2}$ are also $R$-submodular in $N^{*}$.

Proposition 21. If $M_{1}\left(E_{1}, \rho_{1}\right), M_{2}\left(E_{2}, \rho_{2}\right) \in \mathcal{E S M}$ then their direct sum $M_{1} \oplus M_{2} \in$ $\mathcal{E S M}$.

Proof. Let $E=E_{1} \cup E_{2}$, and $M=M_{1} \oplus M_{2}$. Now, let $P_{1}, P_{2}, R \subseteq E, T=E \backslash\left(P_{1} \cup P_{2} \cup R\right)$ and suppose $N \in \mathcal{M F}(M, T)$. By definition, $N=M / X \backslash(T \backslash X)$ for some $X \subseteq T$. If we let, for $i=1,2$,

$$
\begin{gathered}
P_{1 i}=P_{1} \cap E_{i}, \quad P_{2 i}=P_{2} \cap E_{i}, \quad R_{i}=R \cap E_{i}, \\
T_{i}=E_{i} \backslash\left(P_{1} \cup P_{2} \cup R\right), \text { and } N_{i}=M_{i} /\left(X \cap E_{i}\right) \backslash\left(T_{i} \backslash X\right),
\end{gathered}
$$

then it can be checked that $N_{1} \in \mathcal{M F}\left(M_{1}, T_{1}\right), N_{2} \in \mathcal{M F}\left(M_{2}, T_{2}\right)$, and $N=N_{1} \oplus N_{2}$.

As $M_{1}, M_{2} \in \mathcal{E} \mathcal{S} \mathcal{M}$, for $i=1,2$, the sets $P_{1 i}$ and $P_{2 i}$ are $R_{i}$-submodular in $N_{i}$. Hence, from Proposition 13, $P_{1}$ and $P_{2}$ are $R$-submodular in $N$.

We say a matroid $M_{p}$ is a parallel extension of $M(E, \rho)$ if $M_{p} \backslash e=M$, and there exists an $f \in E$ such that $\{e, f\}$ is a circuit in $M_{p}\left[11\right.$, p.155]. Similarly, $M_{s}$ is a series extension of $M(E, \rho)$ if $M_{s} / e=M$, and there is an $f \in E$ such that $\{e, f\}$ is a cocircuit in $M_{s}$.

Proposition 22. Let $M(E, \rho) \in \mathcal{E S M}$.

1. If $M_{p}$ is a parallel extension of $M$, then $M_{p} \in \mathcal{E S \mathcal { M }}$.

2. If $M_{s}$ is a series extension of $M$, then $M_{s} \in \mathcal{E S M}$.

Proof. (1). $\left[M_{p} \in \mathcal{E S \mathcal { M }}\right]$. Let $M_{p} \backslash e=M, E_{p}=E \cup\{e\}$ and $f \in E$ such that $\{e, f\}$ is a circuit in $M_{p}$. Also, let $P_{1}, P_{2}, R \subseteq E_{p}, T=E_{p} \backslash\left(P_{1} \cup P_{2} \cup R\right)$ and $N \in \mathcal{M F}\left(M_{p}, T\right)$. Then, by definition, $N=M_{p} / C \backslash(T \backslash C)$ for some $C \subseteq T$.

We have the following two possible cases.

Case $1\left(e \notin P_{1} \cup P_{2} \cup R\right)$ : Then $e \in T$. There are three possibilities in this case.

Subcase 1a $(e \notin C)$ : Let $T_{e}=T \backslash\{e\}$. As $M=M_{p} \backslash e$, we see that

$$
N=M_{p} / C \backslash(T \backslash C)=M / C \backslash\left(T_{e} \backslash C\right) .
$$

Thus, $N \in \mathcal{M F}\left(M, T_{e}\right)$ and since $M \in \mathcal{E S \mathcal { M }}$, it follows that $P_{1}$ and $P_{2}$ are $R$-submodular in $N$.

Subcase 1b $\left(e \in C, f \notin P_{1} \cup P_{2} \cup R\right)$ : Then $f \in T$. As $\{e, f\}$ is a circuit in $M_{p}$, we know $f$ is a loop in $M_{p} / e$. Let $C_{f}=C \backslash\{f\}$. Then,

$$
N=M_{p} / C \backslash(T \backslash C)=M_{p} / C_{f} \backslash\left(T \backslash C_{f}\right) .
$$


However, as $e$ and $f$ are parallel elements in $M_{p}$ and $f \notin C_{f}$, this is now similar to Subcase 1 a.

Subcase 1c $\left(e \in C, f \in P_{1} \cup P_{2} \cup R\right)$ : Let $C_{e}^{f}=C \cup\{f\} \backslash\{e\}$, and $N_{e}^{f}=M_{p} / C_{e}^{f} \backslash(T \backslash C)$. Then $N_{e}^{f} \in \mathcal{M} \mathcal{F}(M, T \cup\{f\})$.

Also, let $P_{1}^{f}=P_{1} \backslash\{f\}, P_{2}^{f}=P_{2} \backslash\{f\}$ and $R^{f}=R \backslash\{f\}$. Since $M \in \mathcal{E} \mathcal{S M}$, the sets $P_{1}^{f}$ and $P_{2}^{f}$ are $R^{f}$-submodular in $N_{e}^{f}$. But $e$ and $f$ are parallel in $M_{p}$, and so $P_{1}^{f}$ and $P_{2}^{f}$ are also $R^{f}$-submodular in $N=M_{p} / C \backslash(T \backslash C)$. Finally, as $f$ is a loop in $N$, this implies $P_{1}$ and $P_{2}$ are $R$-submodular in $N$ as required in this case.

Case $2\left(e \in P_{1} \cup P_{2} \cup R\right)$ : There are again three different possibilities.

Subcase 2a $\left(f \notin P_{1} \cup P_{2} \cup R\right)$ : If $f \notin P_{1} \cup P_{2} \cup R$, then as $e$ and $f$ are parallel elements in $M_{p}$, this is equivalent to $e \notin P_{1} \cup P_{2} \cup R, f \in P_{1} \cup P_{2} \cup R$, which was handled in Subcases $1 \mathrm{a}$ and $1 \mathrm{c}$ above.

Subcase 2b $\left(e \in R, f \in P_{1} \cup P_{2} \cup R\right)$ : Let $R^{e}=R \backslash\{e\}$. Then, $N \backslash e, N / e \in \mathcal{M F}(M, T)$, and as $M \in \mathcal{E S M}$, we know $P_{1}$ and $P_{2}$ are $R^{e}$-submodular in $N \backslash e$ and $N / e$.

Now, if $f \in R$, since $\{e, f\}$ is a circuit in $N$, we can deduce $P_{1}$ and $P_{2}$ are $R$-submodular in $N$ from Lemma 17.

Also, if $f \in P_{1}$ or $f \in P_{2}$, then $e \in \operatorname{cl}_{N}\left(P_{i}\right)$ for some $i \in\{1,2\}$, and we can use Lemma 16 to obtain $P_{1}$ and $P_{2}$ are $R$-submodular in $N$.

Subcase 2c $\left(e \in P_{1} \cup P_{2}, f \in P_{1} \cup P_{2} \cup R\right)$ : Without loss of generality assume $e \in P_{1}$, and let $P_{1}^{e}=P_{1} \backslash\{e\}$. As $N \backslash e \in \mathcal{M F}(M, T)$ and $M \in \mathcal{E S} \mathcal{M}$, we know $P_{1}^{e}$ and $P_{2}$ are $R$-submodular in $N \backslash e$.

Now, if $f \in P_{1} \cup P_{2}$, then $e \in \mathrm{cl}_{N}\left(P_{1} \cup P_{2}\right)$ and hence, from Lemma 14-1, we deduce $P_{1}$ and $P_{2}$ are $R$-submodular in $N$.

Alternatively, if $f \in R$, as $e$ and $f$ are parallel elements in $N$, the case is analogous to $e \in R$ and $f \in P_{1}$, which was handled in Subcase 2b.

(2). $\left[M_{s} \in \mathcal{E S M}\right]$. Let $M_{s} / e=M$. Then its dual $M_{s}^{*}$ is a parallel extension of $M^{*}$, the dual matroid of $M$, such that $M_{s}^{*} \backslash e=M^{*}[11$, p.155]. We know from Proposition 20 that $M^{*} \in \mathcal{E S M}$, and consequently, from the previous case, its parallel extension $M_{s}^{*} \in \mathcal{E S \mathcal { M }}$. Finally, applying Proposition 20 again, we find $M_{s} \in \mathcal{E} \mathcal{S} \mathcal{M}$.

A parallel class of $M(E, \rho)$ is a maximal set $X \subseteq E$ without loops such that $\rho(X)=1$. A loopless matroid is called simple if all its parallel classes are of size one. Every matroid $M$ has an associated simple matroid $\widetilde{M}$ obtained by deleting its loops and all but one distinguished element from each parallel class of $M[11, \mathrm{p} .52]$. The next result is an immediate consequence of Proposition 22.

Corollary 23. A matroid $M(E, \rho) \in \mathcal{E S M}$ if and only if its associated simple matroid $\widetilde{M} \in \mathcal{E S M}$.

\subsection{Examples and characterization results}

We now identify some well-known classes of matroids that are extended submodular. 
Lemma 24. Let $M(E, \rho)=U_{m, n}$ be a uniform matroid of rank $m$ and size $n$. Then, for any disjoint $P, R \subseteq E$, there exists an $R$-modular bijection $\pi: 2^{R} \rightarrow 2^{R}$ of the ordered $\operatorname{pair}(P, \emptyset)$ in $M$ such that for all $C \subseteq R,|\pi C| \geq|R|-|P|-|C|$.

Proof. When $|R| \leq|P|$, the map $\pi C=C$ will satisfy our requirement.

When $|R|>|P|$, let $k=|R|-|P|$. In this case, first note that when $0 \leq|C|<k / 2$, the map $\pi C=C$ will not satisfy our lemma because then

$$
|\pi C|=|C|<|R|-|P|-|C| .
$$

We instead define $\pi$ as follows.

Let $\mathcal{R}_{i}$ and $\mathcal{R}_{i}^{k}$ be the set of all subsets of $R$ of size $i$ and $k-i$ respectively. We make the following claim.

Claim 25. If $0 \leq i<k / 2$, then $\left|\mathcal{R}_{i}\right| \leq\left|\mathcal{R}_{i}^{k}\right|$.

Proof. Let $r=|R|$ and $\Delta=\left|\mathcal{R}_{i}\right|-\left|\mathcal{R}_{i}^{k}\right|$. Clearly when $|P|=0$, we have $k-i=r-i$, and so $\Delta=0$. When $0<|P|<r$ and $0 \leq i<k / 2$,

$$
\begin{aligned}
\Delta & =\left(\begin{array}{l}
r \\
i
\end{array}\right)-\left(\begin{array}{c}
r \\
k-i
\end{array}\right) \\
& =\frac{r !}{i !(k-i) !}\left(\frac{1}{(k-i+1) \cdots(k-i+r-k)}-\frac{1}{(i+1) \cdots(i+r-k)}\right) .
\end{aligned}
$$

Since $i<k / 2$, for all $j \in \mathbb{Z}$ we have $k-i+j>i+j$, and thus $\Delta<0$.

It follows that for each $0 \leq i<k / 2$ there is an injection $\sigma_{i}: \mathcal{R}_{i} \rightarrow \mathcal{R}_{i}^{k}$. Additionally, for each $0 \leq i<k / 2$ we define a map $\mu_{i}: \mathcal{R}_{i}^{k} \rightarrow \mathcal{R}_{i} \cup \mathcal{R}_{i}^{k}$, which is really the inverse map of $\sigma_{i}$ extended to all elements of $\mathcal{R}_{i}^{k}$ as follows. For all $X \in \mathcal{R}_{i}^{k}$,

$$
\mu_{i}(X)=\left\{\begin{array}{l}
X^{\prime}, \text { if there is a } X^{\prime} \in \mathcal{R}_{i} \text { such that } \sigma_{i} X^{\prime}=X \\
X, \text { otherwise. }
\end{array}\right.
$$

We are finally ready to define the bijection $\pi$ that satisfies our lemma. For all $C \subseteq R$, let

$$
\pi C=\left\{\begin{array}{l}
\sigma_{|C|}(C), \text { if } 0 \leq|C|<k / 2 \\
\mu_{k-|C|}(C), \text { if } k / 2<|C| \leq k \\
C, \text { otherwise }
\end{array}\right.
$$

It remains to show that (13) satisfies our requirements.

When $0 \leq|C|<k / 2$, clearly

$$
|\pi C|=\left|\sigma_{|C|}(C)\right|=k-|C|=|R|-|P|-|C| .
$$

Consequently, in this case $|P \cup C|=|R \backslash \pi C|$ and $|R \backslash C|=|P \cup \pi C|$, and as $M$ is a uniform matroid, this implies $\rho(P \cup C)+\rho(R \backslash C)=\rho(P \cup \pi C)+\rho(R \backslash \pi C)$. 
When $k / 2<|C| \leq k$, we have $|\pi C|=\left|\mu_{k-|C|}(C)\right|$. Hence, from (12) we have two possibilities. First, it can be that

$$
|\pi C|=k-|C|=|R|-|P|-|C|,
$$

and the proof that $\pi$ satisfies our requirements are similar to the previous case. Alternatively, when $k / 2<|C| \leq k$, from (12), we can have

$$
|\pi C|=k-(k-|C|)=|C|>|R|-|P|-|C| .
$$

It also follows trivially in this case that $\rho(P \cup C)+\rho(R \backslash C)=\rho(P \cup \pi C)+\rho(R \backslash \pi C)$.

Finally when $|C|=k / 2$ or $k<|C| \leq|R|$, from (13), we have

$$
|\pi C|=|C| \geq|R|-|P|-|C| \text {. }
$$

Also, trivially, $\rho(P \cup C)+\rho(R \backslash C)=\rho(P \cup \pi C)+\rho(R \backslash \pi C)$, in this case as required.

Lemma 26. Let $M(E, \rho)=U_{m, n}$ be a uniform matroid of rank $m$ and size $n$. Then, for any mutually disjoint $P_{1}, P_{2}, R \subseteq E$, there exists an $R$-submodular bijection $\pi: 2^{R} \rightarrow 2^{R}$ of the pair $\left(P_{1}, P_{2}\right)$ in $M$ such that for all $C \subseteq R,|\pi C| \geq|R|-\left|P_{1}\right|-|C|$.

Proof. We use induction on $\left|P_{2}\right|$. When $\left|P_{2}\right|=0$, the statement follows from Lemma 24 . Let $k \in \mathbb{Z}_{>0}$, and assume the lemma is true whenever $\left|P_{2}\right|<k$.

Suppose $\left|P_{2}\right|=k$, and let $P_{2}^{\prime}=P_{2} \backslash\{p\}$ for some $p \in P_{2}$. By the inductive hypothesis, there exists an $R$-submodular bijection $\pi: 2^{R} \rightarrow 2^{R}$ of $\left(P_{1}, P_{2}^{\prime}\right)$ in $M$ such that for all $C \subseteq$ $R,|\pi C| \geq|R|-\left|P_{1}\right|-|C|$. Equivalently, for all $C \subseteq R$, we have $\left|P_{2}^{\prime} \cup R \backslash \pi C\right| \leq\left|P_{1} \cup P_{2}^{\prime} \cup C\right|$. Since $M$ is uniform, this implies if $p \notin \mathrm{cl}_{M}\left(P_{1} \cup P_{2}^{\prime} \cup C\right)$ then $p \notin \operatorname{cl}_{M}\left(P_{2}^{\prime} \cup R \backslash \pi C\right)$. Hence, for all $C \subseteq R$,

$$
\rho\left(P_{1} \cup P_{2}^{\prime} \cup\{p\} \cup C\right)+\rho(R \backslash C) \leq \rho\left(P_{1} \cup \pi C\right)+\rho\left(P_{2}^{\prime} \cup\{p\} \cup R \backslash \pi C\right) .
$$

Equivalently, $\pi$ is also an $R$-submodular bijection of the pair $\left(P_{1}, P_{2}^{\prime} \cup\{p\}\right)$ in $M$.

We note that it is a curious result of the proofs of Lemmas 24 and 26 that for any mutually disjoint $P_{1}, P_{2}, R \subseteq E$, there exists an $R$-submodular bijection of the pair $\left(P_{1}, P_{2}\right)$ in the uniform matroid $U_{m, n}$ that depends only on the sizes of the sets $P_{2}$ and $R$. In particular, this bijection is entirely independent of the rank $m$ and size $n$ of the matroid.

More importantly, as the class of uniform matroids is minor closed, these results show that all such matroids are extended submodular.

Corollary 27. All uniform matroids are extended submodular.

Chaourar and Oxley [3] showed that the excluded minors for the class of uniform matroids and their series-parallel extensions are $W_{3}, \mathcal{W}_{3}, Q_{6}, P_{6}$ and $R_{6}$. We refer to [11, p.503-504] for a description of the matroids $Q_{6}, P_{6}$ and $R_{6}$. Among these excluded minors, we can see from Example 8 that the matroids $W_{3}$ and $\mathcal{W}_{3}$ are not extended submodular. However, a routine computational verification shows that $Q_{6}, P_{6}, R_{6} \in \mathcal{E S M}[8$, Appendix A]. Following Oxley [11, p.365], for a set $\left\{M_{1}, M_{2}, \ldots\right\}$ of matroids, we use the notation $\operatorname{EX}\left(M_{1}, M_{2}, \ldots\right)$ to denote the class of matroids with no minors isomorphic to any element of $\left\{M_{1}, M_{2}, \ldots\right\}$. Then, from the previous discussion, we get the following result. 
Proposition 28. $E X\left(W_{3}, \mathcal{W}_{3}, Q_{6}, P_{6}, R_{6}\right) \subsetneq \mathcal{E S M}$.

Since all matroids in the excluded minor list of the previous result are of rank three, and the class $\mathcal{E S M}$ is dual closed, we can say the following.

Corollary 29. Every matroid of rank or corank at most two is extended submodular.

Brylawski [2] showed that the series-parallel networks are precisely the class of binary matroids without a minor isomorphic to $W_{3}$. Since $\mathcal{W}_{3}, Q_{6}, P_{6}$ and $R_{6}$ are known to be non-binary matroids, from Proposition 28 and Example 8, we get our next corollary.

Corollary 30. A binary matroid is extended submodular if and only if it is a seriesparallel network.

\section{$4 \quad R$-submodularity and the $Z$-Rayleigh property}

We now show that $R$-submodularity in minors of a matroid enforce non-negativity of corresponding coefficients (explained below in Lemma 33) in its $Z$-Rayleigh difference polynomials defined in (2). We begin by listing some existing results for the class of $Z$-Rayleigh matroids.

Let $M_{1}\left(E_{1}, \rho_{1}\right)$ and $M_{2}\left(E_{2}, \rho_{2}\right)$ be two matroids such that $E_{1} \cap E_{2}=\{e\}$. Then their 2-sum is the matroid $M_{1} \oplus_{2} M_{2}(E, \rho)$ with ground set $E=E_{1} \cup E_{2} \backslash\{e\}$ and for all $X \subseteq E$,

$$
\rho(X)=\left\{\begin{array}{l}
\rho_{1}\left(X \cap E_{1}\right)+\rho_{2}\left(X \cap E_{2}\right)-1, \text { if } e \in \operatorname{cl}_{M_{1}}\left(X \cap E_{1}\right) \cap \operatorname{cl}_{M_{2}}\left(X \cap E_{2}\right) ; \\
\rho_{1}\left(X \cap E_{1}\right)+\rho_{2}\left(X \cap E_{2}\right), \text { otherwise. }
\end{array}\right.
$$

Proposition 31. 1. The class of Z-Rayleigh matroids is closed under duals, minors and 2-sums [16, Lemma 4.1, Theorem 5.8].

2. All uniform matroids are Z-Rayleigh [16, Section 5].

Note that Theorem 5.8 in [16] states that the class of Potts-Rayleigh matroids is closed under 2-sums. Nevertheless, the same argument is readily seen to show that the restricted class of $Z$-Rayleigh matroids is also closed under 2-sums.

It is an immediate consequence of Proposition 31 that all 2-sum extensions of uniform matroids including the series-parallel networks are $Z$-Rayleigh. Our results in the first half of this section shows that uniform matroids and their series-parallel extensions also have the additional property that every coefficient of their $Z$-Rayleigh difference polynomials is non-negative for all $q$ in the interval $0<q \leq 1$.

\subsection{The basic results}

Let $M(E, \rho)$ be a matroid. For any subset $A \subseteq E$, we frequently use the convenient notation $\mathbf{y}^{A}=\prod_{e \in A} y_{e}$ with the convention $\mathbf{y}^{A}=1$ if $A$ is empty. For disjoint sets $A, B \subseteq$ 
$E \backslash\{e, f\}$, we denote the coefficient of the monomial $\mathbf{y}^{A}\left(\mathbf{y}^{B}\right)^{2}$ in $\Delta Z\{e, f\}(M, q ; \mathbf{y})$ by $\left[\mathbf{y}^{A}\left(\mathbf{y}^{B}\right)^{2}\right] \Delta Z\{e, f\}(M, q ; \mathbf{y})$.

The following result is useful to establish the connection between $R$-submodularity of $\{e\}$ and $\{f\}$, and the non-negativity of the coefficients in a $\Delta Z\{e, f\}(M, q ; \mathbf{y})$.

Lemma 32. Let $M(E, \rho)$ be a matroid and $e, f \in E$ be two distinct non-loop elements. Then, for any disjoint pair of sets $R, T \subseteq E \backslash\{e, f\}$ and a bijection $\sigma: 2^{R} \rightarrow 2^{R}$, $\left[\mathbf{y}^{R}\left(\mathbf{y}^{T}\right)^{2}\right] \Delta Z\{e, f\}(M, q ; \mathbf{y})=q^{2} \beta_{R, T}(q)$, where

$$
\beta_{R, T}(q)=\sum_{C \subseteq R}\left(q^{-\rho(\{e\} \cup T \cup \sigma C)-\rho(\{f\} \cup T \cup R \backslash \sigma C)}-q^{-\rho(\{e, f\} \cup T \cup C)-\rho(T \cup R \backslash C)}\right) .
$$

Proof. Let $\bar{E}=E \backslash\{e, f\}$. Recall that for any disjoint $U, V \subseteq E$ and all $A \subseteq E \backslash(V \cup U)$,

$$
\rho_{M / V \backslash U}(A)=\rho(A \cup V)-\rho(V) .
$$

Using (1), (2), (14), we get

$$
\Delta Z\{e, f\}(M, q ; \mathbf{y})=q^{2} \alpha(q ; \mathbf{y})
$$

where

$$
\begin{aligned}
\alpha(q ; \mathbf{y})= & \sum_{(X, Y) \in 2^{\bar{E}} \times 2^{\bar{E}}} q^{-\rho(X \cup\{e\})-\rho(Y \cup\{f\})} \mathbf{y}^{X \cup Y} \mathbf{y}^{X \cap Y} \\
& -\sum_{(W, Z) \in 2^{\bar{E}} \times 2^{\bar{E}}} q^{-\rho(W \cup\{e, f\})-\rho(Z)} \mathbf{y}^{W \cup Z} \mathbf{y}^{W \cap Z} .
\end{aligned}
$$

We can now split each of the two sums in (16) into smaller sums on pairs $(X, Y)$ (and ( $W, Z$ ), respectively) according to $X \cup Y$ and $X \cap Y$ (and $W \cup Z$ and $W \cap Z$, respectively). That is,

$$
\alpha(q ; \mathbf{y})=\sum_{\substack{(A, B) \in 2^{\bar{E}} \times 2^{\bar{E}} \\ A \cap B=\emptyset}} \gamma_{A, B}(q) \cdot \mathbf{y}^{A}\left(\mathbf{y}^{B}\right)^{2},
$$

where

$$
\gamma_{A, B}(q)=\sum_{\substack{(X, Y) \in 2^{\bar{E}} \times 2^{\bar{E}} \\ X \cap Y=B, X \cup Y=A \cup B}} q^{-\rho(X \cup\{e\})-\rho(Y \cup\{f\})}-\sum_{\substack{(W, Z) \in 2^{\bar{E}} \times 2^{\bar{E}} \\ W \cap Z=B, W \cup Z=A \cup B}} q^{-\rho(W \cup\{e, f\})-\rho(Z)} .
$$

From (15) and (17), it is plain that

$$
\left[\mathbf{y}^{R}\left(\mathbf{y}^{T}\right)^{2}\right] \Delta Z\{e, f\}(M, q ; \mathbf{y})=q^{2} \gamma_{R, T}(q) .
$$

Also, note that,

$$
\begin{aligned}
\{(X \cup\{e\}, Y \cup\{f\}):(X, Y) & \left.\in 2^{\bar{E}} \times 2^{\bar{E}}, X \cap Y=T, X \cup Y=R \cup T\right\}= \\
& \{(\{e\} \cup T \cup C,\{f\} \cup T \cup R \backslash C): C \subseteq R\}, \text { and }
\end{aligned}
$$

THE ELECTRONiC Journal of COMBINATORICS 18 (2011), \#P113 


$$
\begin{aligned}
&\left\{(W \cup\{e, f\}, Z):(W, Z) \in 2^{\bar{E}} \times 2^{\bar{E}}, W \cap Z=T, W \cup Z=R \cup T\right\}= \\
&\{(\{e, f\} \cup T \cup C, T \cup R \backslash C): C \subseteq R\} .
\end{aligned}
$$

Hence, given any bijection $\sigma: 2^{R} \rightarrow 2^{R}$, we can rewrite (18) as

$$
\begin{aligned}
\gamma_{R, T}(q) & =\sum_{C \subseteq R}\left(q^{-\rho(\{e\} \cup T \cup \sigma C)-\rho(\{f\} \cup T \cup R \backslash \sigma C)}-q^{-\rho(\{e, f\} \cup T \cup C)-\rho(T \cup R \backslash C)}\right) \\
& =\beta_{R, T}(q) .
\end{aligned}
$$

The result now follows from (19).

One consequence of this observation is particularly relevant for our purposes.

Lemma 33. Let $M(E, \rho)$ be a matroid, e, $f \in E$ be two distinct elements and $\bar{E}=$ $E \backslash\{e, f\}$. Also for any disjoint $R, T \subseteq \bar{E}$, let $\bar{T}=\bar{E} \backslash(T \cup R)$. If the sets $\{e\}$ and $\{f\}$ are $R$-submodular in the minor $N=M / T \backslash \bar{T} \in \mathcal{M F}(M, \bar{E} \backslash R)$, then the coefficient $\left[\mathbf{y}^{R}\left(\mathbf{y}^{T}\right)^{2}\right] \Delta Z\{e, f\}(M, q ; \mathbf{y}) \geq 0$ for all $q$ in the interval $0<q \leq 1$.

Furthermore, $\left[\mathbf{y}^{R}\left(\mathbf{y}^{T}\right)^{2}\right] \Delta Z\{e, f\}(M, q ; \mathbf{y})=0$ for all $q$ in the interval $0<q \leq 1$ if and only if the sets $\{e\}$ and $\{f\}$ are $R$-modular in $N$.

Proof. It is easy to check that if $e$ or $f$ is a loop in $M$ then $\Delta Z\{e, f\}(M, q ; \mathbf{y})=0$, and the lemma is vacuously true. Henceforth we assume that neither $e$ nor $f$ is a loop in $M$.

Let $\pi: 2^{R} \rightarrow 2^{R}$ be an $R$-submodular bijection for the pair $(\{e\},\{f\})$ in $N=M / T \backslash \bar{T}$. Then, we know from Lemma $32,\left[\mathbf{y}^{R}\left(\mathbf{y}^{T}\right)^{2}\right] \Delta Z\{e, f\}(M, q ; \mathbf{y})=q^{2} \beta_{R, T}(q)$, where

$$
\beta_{R, T}(q)=\sum_{C \subseteq R}\left(q^{-\rho(\{e\} \cup T \cup \pi C)-\rho(\{f\} \cup T \cup R \backslash \pi C)}-q^{-\rho(\{e, f\} \cup T \cup C)-\rho(T \cup R \backslash C)}\right) .
$$

Since $\rho_{N}(A)=\rho(A \cup T)-\rho(T)$ for all $A \subseteq R \cup\{e, f\}$ and $\pi$ is an $R$-submodular bijection, we have for all $C \subseteq R$,

$$
\rho(\{e, f\} \cup T \cup C)+\rho(T \cup R \backslash C) \leq \rho(\{e\} \cup T \cup \pi C)+\rho(\{f\} \cup T \cup R \backslash \pi C) .
$$

Hence, each term of the sum in (20) is at least zero whenever $0<q \leq 1$, which gives $\left[\mathbf{y}^{R}\left(\mathbf{y}^{T}\right)^{2}\right] \Delta Z\{e, f\}(M, q ; \mathbf{y}) \geq 0$.

However, if $\pi$ is an $R$-modular bijection of $(\{e\},\{f\})$ in $N$, then for all $C \subseteq R$,

$$
\rho(\{e, f\} \cup T \cup C)+\rho(T \cup R \backslash C)=\rho(\{e\} \cup T \cup \pi C)+\rho(\{f\} \cup T \cup R \backslash \pi C),
$$

and again from (20) it follows that $\left[\mathbf{y}^{R}\left(\mathbf{y}^{T}\right)^{2}\right] \Delta Z\{e, f\}(M, q ; \mathbf{y})=0$ whenever $0<q \leq 1$.

Conversely, if $\left[\mathbf{y}^{R}\left(\mathbf{y}^{T}\right)^{2}\right] \Delta Z\{e, f\}(M, q ; \mathbf{y})=0$ whenever $0<q \leq 1$, from Lemma 32, we must have $\beta_{R, T}(q)=0$ whenever $0<q \leq 1$. This implies $\beta_{R, T}(q)$ is zero everywhere, for otherwise, as a polynomial in $q^{-1}$, it can only have finitely many roots for $q$ in the interval $0<q \leq 1$. That is, there exists a bijection $\pi: 2^{R} \rightarrow 2^{R}$ such that every term of the sum in (20) is zero, which implies for all $C \subseteq R$,

$$
\rho(\{e, f\} \cup T \cup C)+\rho(T \cup R \backslash C)=\rho(\{e\} \cup T \cup \pi C)+\rho(\{f\} \cup T \cup R \backslash \pi C),
$$

In other words, $\pi$ is an $R$-modular bijection of $(\{e\},\{f\})$ in $N$. 
It is worth noting that while $R$-submodularity of sets $\{e\}$ and $\{f\}$ in a minor is sufficient for non-negativity of the corresponding coefficient in the $Z$-Rayleigh difference polynomial, we do not know if it is also a necessary condition.

Following Wagner [16], we use the notation $Z_{1}(\mathbf{y}) \gg Z_{2}(\mathbf{y})$ for two multivariate polynomials $Z_{1}(\mathbf{y}), Z_{2}(\mathbf{y})$, if $Z_{1}(\mathbf{y})-Z_{2}(\mathbf{y})$ has only non-negative coefficients. The next result is an immediate consequence of Lemma 33, and is often useful in identifying cases where $\Delta Z\{e, f\}(M, q ; \mathbf{y}) \gg 0$ for all $q$ in the interval $0<q \leq 1$.

Theorem 34. Let $M(E, \rho)$ be a matroid, $e, f \in E$ be distinct elements and $\bar{E}=E \backslash\{e, f\}$. If for all $R \subseteq \bar{E}$ and minors $N \in \mathcal{M F}(M, \bar{E} \backslash R)$, the sets $\{e\}$ and $\{f\}$ are $R$-submodular in $N$, then $\Delta Z\{e, f\}(M, q ; \mathbf{y}) \gg 0$ whenever $0<q \leq 1$.

In particular, all extended submodular matroids are also $Z$-Rayleigh because the $R$-submodularity conditions of Theorem 34 are satisfied by definition, for all distinct elements $e, f$ in its ground set.

Proposition 35. If $M(E, \rho) \in \mathcal{E S M}$ then $M$ is $Z$-Rayleigh, and for all distinct $e, f \in E$, $\Delta Z\{e, f\}(M, q ; \mathbf{y}) \gg 0$ whenever $0<q \leq 1$.

\subsection{Beyond extended submodular matroids}

We next apply $R$-submodularity to obtain some results on the $Z$-Rayleigh properties of matroids that are not extended submodular. We begin with the following result which shows that a Theorem proved by Semple and Welsh [12, Theorem 4.2] for the I-Rayleigh property also extends to the $Z$-Rayleigh property.

Theorem 36. Let $M(E, \rho)=M(G)$ be the cycle matroid of a graph $G$. Also let $e, f$ be distinct edges in $E$, and $\bar{E}=E \backslash\{e, f\}$. If $\left[\mathbf{y}^{R}\left(\mathbf{y}^{T}\right)^{2}\right] \Delta Z\{e, f\}(M, q ; \mathbf{y})<0$ for some disjoint $R, T \subseteq \bar{E}, 0<q \leq 1$ and $\mathbf{y}>0$, then the graph $G / T \backslash \bar{T}$ contains a $K_{4}$ minor, where $\bar{T}=\bar{E} \backslash(R \cup T)$.

Proof. Let $H=G / T \backslash \bar{T}$. Then, from Lemma 33 we know $\{e\}$ and $\{f\}$ are not $R$ submodular in $M(H)$, and thus, from Corollary $30, H$ has a $K_{4}$ minor.

Wagner [16, Conjecture 5.4] proposed a stronger conjecture for the $I$-Rayleigh property of graphic matroids requiring the edges $e$ and $f$ in the above result to also be non-adjacent edges of the $K_{4}$ minor in $G$. It is likely that an analogous result is true for the $Z$-Rayleigh property of graphic matroids, and we offer the following conjecture.

Conjecture 37. Let $M(E, \rho)=M(G)$ be the cycle matroid of a graph $G$. Also let e, $f$ be distinct edges in $E$, and $\bar{E}=E \backslash\{e, f\}$. If $\left[\mathbf{y}^{R}\left(\mathbf{y}^{T}\right)^{2}\right] \Delta Z\{e, f\}(M, q ; \mathbf{y})<0$ for some disjoint $R, T \subseteq \bar{E}, 0<q \leq 1$ and $\mathbf{y}>0$, then the graph $G / T \backslash \bar{T}$ contains a $K_{4}$ minor with $e$ and $f$ as non-adjacent edges, where $\bar{T}=\bar{E} \backslash(R \cup T)$.

We next give a characterization of minor-minimal non- $Z$-Rayleigh matroids using $R$ submodularity. 
Lemma 38. Let $M(E, \rho)$ be a matroid with distinct elements e, $f, g \in E$, and let $\bar{E}=$ $E \backslash\{e, f, g\}$. If for all $R \subseteq \bar{E}$, the sets $\{e\}$ and $\{f\}$ are $R \cup\{g\}$-submodular in every minor $N \in \mathcal{M F}(M, \bar{E} \backslash R)$, then

$$
\Delta Z\{e, f\}(M, q ; \mathbf{y}) \gg \Delta Z\{e, f\}(M \backslash g, q ; \mathbf{y})+y_{g}^{2} \Delta Z\{e, f\}(M / g, q ; \mathbf{y}),
$$

whenever $0<q \leq 1$.

Proof. Applying a routine manipulation, we can write

$$
\Delta Z\{e, f\}(M, q ; \mathbf{y})=\Delta Z\{e, f\}(M \backslash g, q ; \mathbf{y})+y_{g}^{2} \Delta Z\{e, f\}(M / g, q ; \mathbf{y})+\Delta_{g}(\mathbf{y}),
$$

where $\Delta_{g}(\mathbf{y})$ contains precisely those monomials of $\Delta Z\{e, f\}(M, q ; \mathbf{y})$ that are of the form $\mathbf{y}^{R \cup\{g\}}\left(\mathbf{y}^{T}\right)^{2}$ for disjoint $R, T \subseteq \bar{E}$. It follows then from Lemma 33 that $\Delta_{g}(\mathbf{y}) \gg 0$ for all $q$ in the interval $0<q \leq 1$, and hence the result.

Corollary 39. Let $M(E, \rho)$ be a minor-minimal matroid that is not Z-Rayleigh, with $e, f \in E$ such that $\Delta Z\{e, f\}(M, q ; \mathbf{y})<0$ for some $q$ in the interval $0<q \leq 1$ and $\mathbf{y}>0$. Then for every $g \in E \backslash\{e, f\}$ there exists an $R \subseteq E_{g}$ and a minor $N \in \mathcal{M F}\left(M, E_{g} \backslash R\right)$ such that $\{e\}$ and $\{f\}$ are not $R \cup\{g\}$-submodular in $N$, where $E_{g}=E \backslash\{e, f, g\}$.

Proof. Suppose for a contradiction that there exists a $g \in E \backslash\{e, f\}$ such that for all $R \subseteq E_{g}$, the sets $\{e\}$ and $\{f\}$ are $R \cup\{g\}$-submodular in every minor $N \in \mathcal{M F}\left(M, E_{g} \backslash R\right)$. Then, by Lemma 38

$$
\Delta Z\{e, f\}(M, q ; \mathbf{y}) \gg \Delta Z\{e, f\}(M \backslash g, q ; \mathbf{y})+y_{g}^{2} \Delta Z\{e, f\}(M / g, q ; \mathbf{y}) .
$$

However, since $M$ is a minor-minimal non- $Z$-Rayleigh matroid, both $M \backslash g$ and $M / g$ are $Z$-Rayleigh, which implies $\Delta Z\{e, f\}(M, q ; \mathbf{y}) \geq 0$ whenever $0<q \leq 1$ and $\mathbf{y}>0$, a contradiction.

In particular, this leads us to a characterization of minor-minimal non-Z-Rayleigh matroids that is similar to a result by Wagner [15, Proposition 3.2] for $B$-Rayleigh matroids. We first need the following $R$-submodularity result, which despite notational differences, is really an extension of the argument used in the proof of [15, Lemma 3.1].

Lemma 40. Let $M(E, \rho)$ be a simple and cosimple matroid with distinct elements e, $f, g \in$ $E$ such that $\{e, f, g\}$ is a circuit or a cocircuit in $M$. Then for all $R \subseteq E \backslash\{e, f, g\}$, the sets $\{e\}$ and $\{f\}$ are $R \cup\{g\}$-submodular in $M$.

Proof. Let $\bar{E}=E \backslash\{e, f, g\}$ and $R \subseteq \bar{E}$. We consider the two cases separately.

Case $1(\{e, f, g\}$ is a circuit in $M)$ : We define the $R \cup\{g\}$-submodular bijection of $(\{e\},\{f\})$ in $M$ as follows. For all $C \subseteq R$, let

$$
\begin{aligned}
\pi C & =\left\{\begin{array}{l}
R \backslash C, \text { if } e, g \notin \mathrm{cl}_{M}(R \backslash C) ; \\
\{g\} \cup C, \text { otherwise },
\end{array}\right. \\
\pi(\{g\} \cup C) & =\left\{\begin{array}{l}
\{g\} \cup C, \text { if } e, g \notin \mathrm{cl}_{M}(R \backslash C) ; \\
R \backslash C, \text { otherwise }
\end{array}\right.
\end{aligned}
$$

THE ELECTRONiC JOURNAL OF COMBINATORICS 18 (2011), \#P113 
We first show that $\pi: 2^{\{g\} \cup R} \rightarrow 2^{\{g\} \cup R}$ is a bijection.

Suppose there exist $C_{1}, C_{2} \subseteq R$ such that $\pi C_{1}=\pi C_{2}$ or $\pi\left(\{g\} \cup C_{1}\right)=\pi\left(\{g\} \cup C_{2}\right)$, then since $g \notin R$, it is easy to see from (21a) and (21b) that $C_{1}=C_{2}$.

Alternatively, suppose there exist $C_{1}, C_{2}$ such that $\pi C_{1}=\pi\left(\{g\} \cup C_{2}\right)$. Then, if $g \in \pi C_{1}$, from (21a) and (21b), we get $C_{1}=C_{2}$, at least one of $e, g \in \mathrm{cl}_{M}\left(R \backslash C_{1}\right)$, and both $e, g \notin \mathrm{cl}_{M}\left(R \backslash C_{2}\right)$, which is a contradiction. A very similar argument gives a contradiction when $g \notin \pi C_{1}$. Hence there cannot be $C_{1}, C_{2} \subseteq R$ such that $\pi C_{1}=\pi\left(\{g\} \cup C_{2}\right)$, and $\pi$ is a bijection.

It remains to show that $\pi$ is a $R \cup\{g\}$-submodular bijection of $(\{e\},\{f\})$ in $M$. As $\{e, f, g\}$ is a circuit in $M$, we have for all $C \subseteq R$,

$$
\rho(\{e, f, g\} \cup C)=\rho(\{e, f\} \cup C)=\rho(\{e, g\} \cup C)=\rho(\{f, g\} \cup C) .
$$

We have the following three possibilities for a subset $C \subseteq R$.

Subcase 1a $\left(g \in \mathrm{cl}_{M}(R \backslash C)\right)$ : Then $\rho(\{g\} \cup R \backslash C) \leq \rho(\{f\} \cup R \backslash C)$. Hence, from (21a), (21b) and (22),

$$
\begin{aligned}
\rho(\{e, f\} \cup C)+\rho(\{g\} \cup R \backslash C) & \leq \rho(\{e, g\} \cup C)+\rho(\{f\} \cup R \backslash C), \\
& =\rho(\{e\} \cup \pi C)+\rho(\{f, g\} \cup R \backslash \pi C), \text { and } \\
\rho(\{e, f, g\} \cup C)+\rho(R \backslash C) & \leq \rho(\{e\} \cup R \backslash C)+\rho(\{f, g\} \cup C) \\
& =\rho(\{e\} \cup \pi(\{g\} \cup C))+\rho(\{f, g\} \cup R \backslash \pi(\{g\} \cup C)) .
\end{aligned}
$$

Subcase $1 \mathrm{~b}\left(e, g \notin \mathrm{cl}_{M}(R \backslash C)\right)$ : Then $\rho(\{e\} \cup R \backslash C)=\rho(\{g\} \cup R \backslash C)$. Thus, from (21a), (21b) and (22),

$$
\begin{aligned}
\rho(\{e, f\} \cup C)+\rho(\{g\} \cup R \backslash C) & =\rho(\{e\} \cup R \backslash C)+\rho(\{f, g\} \cup C) \\
& =\rho(\{e\} \cup \pi C)+\rho(\{f, g\} \cup R \backslash \pi C), \text { and } \\
\rho(\{e, f, g\} \cup C)+\rho(R \backslash C) & \leq \rho(\{e, g\} \cup C)+\rho(\{f\} \cup R \backslash C) \\
& =\rho(\{e\} \cup \pi(\{g\} \cup C))+\rho(\{f, g\} \cup R \backslash \pi(\{g\} \cup C)) .
\end{aligned}
$$

Subcase 1c $\left(e \in \mathrm{cl}_{M}(R \backslash C), g \notin \mathrm{cl}_{M}(R \backslash C)\right)$ : Then observe that $f \notin \mathrm{cl}_{M}(R \backslash C)$. For otherwise, if both $e, f \in \mathrm{cl}_{M}(R \backslash C)$ then

$$
g \in \operatorname{cl}_{M}(\{e, f\} \cup(R \backslash C))=\operatorname{cl}_{M}(R \backslash C),
$$

which is a contradiction. So we have $\rho(\{g\} \cup R \backslash C)=\rho(\{f\} \cup R \backslash C)$, and from (21a), (21b) and (22), it follows that

$$
\begin{aligned}
\rho(\{e, f\} \cup C)+\rho(\{g\} \cup R \backslash C) & =\rho(\{e, g\} \cup C)+\rho(\{f\} \cup R \backslash C), \\
& =\rho(\{e\} \cup \pi C)+\rho(\{f, g\} \cup R \backslash \pi C), \text { and } \\
\rho(\{e, f, g\} \cup C)+\rho(R \backslash C) & \leq \rho(\{e\} \cup R \backslash C)+\rho(\{f, g\} \cup C) \\
& =\rho(\{e\} \cup \pi(\{g\} \cup C))+\rho(\{f, g\} \cup R \backslash \pi(\{g\} \cup C)) .
\end{aligned}
$$


Hence, $\pi$ is a $\{g\} \cup R$-submodular bijection of $(\{e\},\{f\})$ in $M$ in this case.

Case $2(\{e, f, g\}$ is a cocircuit in $M)$ : Consider the matroid $N^{*}=M^{*} /(\bar{E} \backslash R)$. Clearly, $\{e, f, g\}$ is a circuit in $N^{*}$, and hence by Case 1 , we know $\{e\}$ and $\{f\}$ are $R \cup\{g\}$ submodular in $N^{*}$. Now, applying Proposition 11, we get $\{e\}$ and $\{f\}$ are also $R \cup\{g\}$ submodular in $M$ as required.

Corollary 41. Let $M(E, \rho)$ be a simple and cosimple matroid with distinct elements $e, f, g \in E$ such that $\{e, f, g\}$ is a circuit or a cocircuit in $M$, and let $\bar{E}=E \backslash\{e, f, g\}$. Then for all $R \subseteq \bar{E}$, the sets $\{e\}$ and $\{f\}$ are $R \cup\{g\}$-submodular in every minor $N \in$ $\mathcal{M F}(M, \bar{E} \backslash R)$.

Proof. Clearly, for all $R \subseteq \bar{E},\{e, f, g\}$ is a circuit or a cocircuit in every minor $N \in$ $\mathcal{M F}(M, \bar{E} \backslash R)$, and the result follows from Lemma 40.

We can now obtain the following characterization for a minor-minimal non- $Z$-Rayleigh matroid analogous to Proposition 3.2 in [15] for minon-minimal non- $B$-Rayleigh matroids.

Corollary 42. Let $M(E, \rho)$ be a minor-minimal matroid that is not $Z$-Rayleigh. Then, for all $e, f \in E$ such that $\Delta Z\{e, f\}(M, q ; \mathbf{y})<0$ for some $q$ in the interval $0<q \leq 1$ and $\mathbf{y}>0$, the set $\{e, f\}$ is independent and closed in both $M$ and $M^{*}$.

Proof. From Proposition 31-1, we know that if $M$ is a minor-minimal non- $Z$-Rayleigh matroid, then $M$ is both simple and cosimple. Hence $\{e, f\}$ is independent in both $M$ and $M^{*}$.

Let $e, f \in E$ be such that $\Delta Z\{e, f\}(M, q ; \mathbf{y})<0$ for some $q$ in the interval $0<q \leq 1$ and $\mathbf{y}>0$. To show that $\{e, f\}$ is closed in $M$ and $M^{*}$, suppose for a contradiction that there exists an element $g \in E \backslash\{e, f\}$ such that $\{e, f, g\}$ is a circuit or a cocircuit in $M$. However, applying Corollaries 41 and 39, we note that this contradicts our choice of $e$ and $f$. Thus $\{e, f\}$ must be closed in $M$ and $M^{*}$.

\subsection{Rank-three matroids}

When considering matroids of rank three, Lemma 38 gives us the following useful reduction lemma (see [15, Lemma 3.3] for an analogous result on $B$-Rayleigh matroids).

Corollary 43. Let $M(E, \rho)$ be a rank-three simple and cosimple matroid with distinct elements e, $f, g \in E$, and let $\bar{E}=E \backslash\{e, f, g\}$. If for all $R \subseteq \bar{E}$, the sets $\{e\}$ and $\{f\}$ are $R \cup\{g\}$-submodular in every minor $N \in \mathcal{M F}(M, \bar{E} \backslash R)$, then $\Delta Z\{e, f\}(M, q ; \mathbf{y}) \gg$ $\Delta Z\{e, f\}(M \backslash g, q ; \mathbf{y})$ for all $q$ in the interval $0<q \leq 1$.

Proof. From Lemma 38, we have

$$
\Delta Z\{e, f\}(M, q ; \mathbf{y}) \gg \Delta Z\{e, f\}(M \backslash g, q ; \mathbf{y})+y_{g}^{2} \Delta Z\{e, f\}(M / g, q ; \mathbf{y}),
$$

whenever $0<q \leq 1$. Additionally, when $M$ is of rank three, we know $M / g$ is of rank two, and so by Corollary 29 and Proposition 35, $\Delta Z\{e, f\}(M / g, q ; \mathbf{y}) \gg 0$ whenever $0<q \leq 1$. 

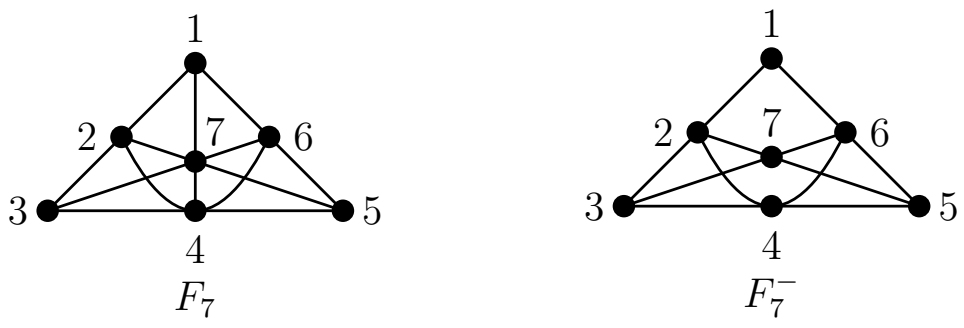

Figure 3: The Fano and non-Fano matroids

We are now ready to show that some important rank three matroids are $Z$-Rayleigh.

Theorem 44. The matroids $W_{3}, \mathcal{W}_{3}, F_{7}$ and $F_{7}^{-}$are $Z$-Rayleigh.

Proof. We look at each of the matroids individually.

Case $M=W_{3}$ : Using symmetry in Figure 1, it is enough to check $\Delta Z\{1,2\}(M, q ; \mathbf{y})$ and $\Delta Z\{1,4\}(M, q ; \mathbf{y})$ are non-negative whenever $0<q \leq 1$ and $\mathbf{y}>0$.

Since $\{1,2,3\}$ is a circuit, from Corollaries 41 and 43 , we have $\Delta Z\{1,2\}(M, q ; \mathbf{y}) \gg$ $\Delta Z\{1,2\}(M \backslash 3, q ; \mathbf{y}) \gg 0$ whenever $0<q \leq 1$, as $M \backslash 3 \in \mathcal{E S} \mathcal{M}$ by Proposition 28 .

From Proposition 7 and Lemma 33, the only monomial whose coefficient can be nonnegative in $\Delta Z\{1,4\}(M, q ; \mathbf{y})$ for some $q$ in the interval $0<q \leq 1$ is $y_{2} y_{3} y_{5} y_{6}$. Hence, we can write

$$
\Delta Z\{1,4\}(M, q ; \mathbf{y})=A_{1}(q) y_{2} y_{3} y_{5} y_{6}+A_{2}(q) y_{2}^{2} y_{5}^{2}+A_{3}(q) y_{3}^{2} y_{6}^{2}+\Delta_{1}(\mathbf{y}),
$$

where $A_{1}(q), A_{2}(q)$ and $A_{3}(q)$ are the coefficients of the monomials $y_{2} y_{3} y_{5} y_{6}, y_{2}^{2} y_{5}^{2}$ and $y_{3}^{2} y_{6}^{2}$ in $\Delta Z\{1,4\}(M, q ; \mathbf{y})$ respectively, and $\Delta_{1}(\mathbf{y}) \gg 0$ whenever $0<q \leq 1$.

Using the identity map between columns two and three of Table 1 as the bijection in Lemma 32 , we obtain

$$
A_{1}(q)=2\left(q^{-3}-q^{-4}\right)+3\left(q^{-3}-q^{-2}\right)+\left(q^{-2}-q^{-1}\right) .
$$

Also, using Lemma 32, it is straightforward to get

$$
A_{2}(q)=A_{3}(q)=q^{-4}-q^{-3} .
$$

Using (24) and (25) in (23), we get

$$
\begin{aligned}
\Delta Z\{1,4\}(M, q ; \mathbf{y})= & \left(q^{-4}-q^{-3}\right)\left(y_{2} y_{5}-y_{3} y_{6}\right)^{2}+ \\
& \left(3\left(q^{-3}-q^{-2}\right)+\left(q^{-2}-q^{-1}\right)\right) y_{2} y_{3} y_{5} y_{6}+\Delta_{1}(\mathbf{y}),
\end{aligned}
$$

which is non-negative whenever $0<q \leq 1$ and $\mathbf{y}>0$. Hence, $W_{3}$ is $Z$-Rayleigh.

Case $M=\mathcal{W}_{3}$ : Using symmetry in Figure 1 , it is enough to check $\Delta Z\{1,2\}(M, q ; \mathbf{y})$, $\Delta Z\{2,6\}(M, q ; \mathbf{y})$ and $\Delta Z\{1,4\}(M, q ; \mathbf{y})$ are non-negative whenever $0<q \leq 1$ and $\mathbf{y}>0$. 
As $\{1,2,3\}$ is a circuit, from Corollaries 41 and 43 , we get $\Delta Z\{1,2\}(M, q ; \mathbf{y}) \gg$ $\Delta Z\{1,2\}(M \backslash 3, q ; \mathbf{y}) \gg 0$ because $M \backslash 3 \in \mathcal{E S M}$ by Proposition 28.

Also $\{1,2,6\}$ is a cocircuit in $M$, and again from Corollaries 41 and 43 , we get $\Delta Z\{2,6\}(M, q ; \mathbf{y}) \gg \Delta Z\{2,6\}(M \backslash 1, q ; \mathbf{y}) \gg 0$ whenever $0<q \leq 1$, as $M \backslash 1 \in \mathcal{E S M}$ by Proposition 28.

Lastly, from Proposition 7 and Lemma 33, the only monomial whose coefficient can be non-negative in $\Delta Z\{1,4\}(M, q ; \mathbf{y})$ is $y_{2} y_{3} y_{5} y_{6}$. Hence,

$$
\Delta Z\{1,4\}(M, q ; \mathbf{y})=A_{4}(q) y_{2} y_{3} y_{5} y_{6}+A_{5}(q) y_{2}^{2} y_{5}^{2}+A_{6}(q) y_{3}^{2} y_{6}^{2}+\Delta_{2}(\mathbf{y}),
$$

where $A_{4}(q), A_{5}(q)$ and $A_{6}(q)$ are the coefficients of the monomials $y_{2} y_{3} y_{5} y_{6}, y_{2}^{2} y_{5}^{2}$ and $y_{3}^{2} y_{6}^{2}$ in $\Delta Z\{1,4\}(M, q ; \mathbf{y})$ respectively, and $\Delta_{2}(\mathbf{y}) \gg 0$ whenever $0<q \leq 1$.

Using the identity map between columns four and five in Table 1 as the bijection in Lemma 32, we get

$$
A_{4}(q)=\left(q^{-3}-q^{-4}\right)+3\left(q^{-3}-q^{-2}\right)+\left(q^{-2}-q^{-1}\right) .
$$

Again using Lemma 32 we can get,

$$
A_{5}(q)=A_{6}(q)=q^{-4}-q^{-3}
$$

Using (27) and (28) in (26), we find

$$
\begin{aligned}
\Delta Z\{1,4\}(M, q ; \mathbf{y})= & \left(q^{-4}-q^{-3}\right)\left(y_{2} y_{5}-y_{3} y_{6}\right)^{2}+ \\
& \left(\left(q^{-4}-q^{-3}\right)+3\left(q^{-3}-q^{-2}\right)+\left(q^{-2}-q^{-1}\right)\right) y_{2} y_{3} y_{5} y_{6}+\Delta_{2}(\mathbf{y}),
\end{aligned}
$$

which is non-negative whenever $0<q \leq 1$ and $\mathbf{y}>0$. Thus $\mathcal{W}_{3}$ is $Z$-Rayleigh.

Case $M=F_{7}$ : Using symmetry in Figure 3 , it is enough to check $\Delta Z\{1,4\}(M, q ; \mathbf{y})$ is non-negative whenever $0<q \leq 1$ and $\mathbf{y}>0$. Since $\{1,4,7\}$ is a circuit, from Corollaries 41 and $43, \Delta Z\{1,4\}(M, q ; \mathbf{y}) \gg \Delta Z\{1,4\}(M \backslash 7, q ; \mathbf{y})$. But $M \backslash 7=W_{3}$, and we showed in a previous case $\Delta Z\{1,4\}(M \backslash 7, q ; \mathbf{y}) \geq 0$ whenever $0<q \leq 1$ and $\mathbf{y}>0$. Hence, $F_{7}$ is $Z$-Rayleigh.

Case $M=F_{7}^{-}$: Using symmetry in Figure 3, it is enough to check $\Delta Z\{1,2\}(M, q ; \mathbf{y})$ and $\Delta Z\{1,4\}(M, q ; \mathbf{y})$ are non-negative whenever $0<q \leq 1$ and $\mathbf{y}>0$.

Since $\{1,2,3\}$ is a circuit, from Corollaries 41 and 43 , we know $\Delta Z\{1,2\}(M, q ; \mathbf{y}) \gg$ $\Delta Z\{1,2\}(M \backslash 3, q ; \mathbf{y})$. However, $M \backslash 3$ is isomorphic to $\mathcal{W}_{3}$, and hence from a previous case, $\Delta Z\{1,2\}(M, q ; \mathbf{y})$ is non-negative whenever $0<q \leq 1$ and $\mathbf{y}>0$.

For $\Delta Z\{1,4\}(M, q ; \mathbf{y})$, we first show that $\{1\}$ and $\{4\}$ are $R \cup\{7\}$-submodular in $N$ for all $R \subseteq \bar{E}$ and minors $N \in \mathcal{M F}(M, \bar{E} \backslash R)$, where $\bar{E}=\{2,3,5,6\}$. First, it can be computationally checked that $\{1\}$ and $\{4\}$ are $\{2,3,5,6,7\}$-submodular in $F_{7}^{-}[8$, Appendix B]. Also $\{1,4,5\}$ is a cocircuit in $M \backslash 2$. Hence, from Lemma 40, $\{1\}$ and $\{4\}$ are $\{3,5,6,7\}$-submodular in $M \backslash 2$. A similar argument shows that for each $e \in \bar{E}$, the sets $\{1\}$ and $\{4\}$ are $R \cup\{7\}$-submodular in $M \backslash e$, where $R=\bar{E} \backslash\{e\}$. Since for every $e \in \bar{E}$, $M / e$ is a rank-two matroid, by Corollary $29,\{1\}$ and $\{4\}$ are also $R \cup\{7\}$-submodular in $M / e$, where $R=\bar{E} \backslash\{e\}$. Together with Proposition 7, these observations imply that $\{1\}$ and $\{4\}$ are $R \cup\{7\}$-submodular in $N$ for all $R \subseteq \bar{E}$ and $N \in \mathcal{M F}(M, \bar{E} \backslash R)$. 
Hence, using Corollary $43, \Delta Z\{1,4\}(M, q ; \mathbf{y}) \gg \Delta Z\{1,4\}(M \backslash 7, q ; \mathbf{y})$ for all $q$ in the interval $0<q \leq 1$. But $M \backslash 7=W_{3}$, and thus we get $\Delta Z\{1,4\}(M, q ; \mathbf{y}) \geq 0$ whenever $0<q \leq 1$ and $\mathbf{y}>0$ from a previous case, and $F_{7}^{-}$is $Z$-Rayleigh.

From Proposition 31-1, we know that the class of $Z$-Rayleigh matroids is closed under duality, and we get the following corollary.

Corollary 45. The matroids $F_{7}^{*}$ and $\left(F_{7}^{-}\right)^{*}$ are $Z$-Rayleigh.

\section{$5 \quad$ Summary and open problems}

We introduced $R$-submodularity as an extension of rank submodularity of matroids. While some instances of $R$-submodularity are known for all matroids, we also showed that there exists a class of well-behaved matroids with respect to $R$-submodularity, namely the class of extended submodular matroids, $\mathcal{E} \mathcal{S M}$. Also the class of binary extended submodular matroids is identical to series-parallel networks. Yet a complete characterization of extended submodular matroids remains unresolved and is our first open problem. A positive step towards resolving this problem will be to find example matroids (if any) that are not extended submodular and without a $W_{3}$ or a $\mathcal{W}_{3}$ minor. The related question of whether the class $\mathcal{E} \mathcal{S M}$ is closed under 2-sums is also unresolved.

As discussed in Section 4, the $R$-submodularity property has important consequences for the Rayleigh properties of matroids, and deserves further study. We suggest that $R$ submodularity can be a particularly effective tool in establishing the Rayleigh property for cases where the Rayleigh difference polynomials are expected to have only non-negative coefficients. As an example, we offer the following conjecture for graphs.

Conjecture 46. Let $M(G)$ be the cycle matroid of a graph $G$ with edge set $E$. If $e, f \in E$ are two distinct incident edges in $G$ then for all $R \subseteq E \backslash\{e, f\}$, the sets $\{e\}$ and $\{f\}$ are $R$-submodular in $M(G)$.

Since the class of graphic matroids is minor closed and the incidence property is preserved in minors, if true, this conjecture will show that $\Delta Z\{e, f\}(M, q ; \mathbf{y}) \gg 0$ for all $q$ in $0<q \leq 1$ when $M$ is graphic and $e, f$ are incident edges of its graph representation. We note that it is a consequence of Corollary 42 that a minor-minimal counterexample of Conjecture 46 cannot have triangles including the edges $e$ and $f$, and thus is not a complete graph.

We also illustrate in Theorem 44 that even in cases where $\Delta Z\{e, f\}(M, q ; \mathbf{y})$ contains a few negative coefficients for some $q$ in the interval $0<q \leq 1, R$-submodularity can significantly reduce the computation required to verify if $\Delta Z\{e, f\}(M, q ; \mathbf{y}) \geq 0$ whenever $0<q \leq 1$ and $\mathbf{y}>0$. Based on our empirical results with a few different rank-three matroids, we also pose the following open problem that is a significant strengthening of Theorem 1.1 in [15].

Conjecture 47. Every matroid of rank or corank at most three is Z-Rayleigh. 
Lastly, an important unanswered question in the study of the various Rayleigh properties of matroids is if their respective classes are different from one another. For example, while it is likely there are matroids that are $B$-Rayleigh but not $Z$-Rayleigh, finding an example of such a matroid still remains open.

\section{Acknowledgements}

I am thankful to Alan Sokal for engaging discussions on the multivariate Tutte polynomial and the various Rayleigh properties of matroids that stimulated much of the research discussed in this paper. He also pointed out some relevant previous results, conjectured a stronger form of Proposition 28 and offered helpful advice on improving the notations. I would also like to thank Steve Noble for helpful insights on $R$-submodularity, and Graham Farr and David Wagner for advice and critical feedback on early drafts of this paper.

\section{References}

[1] A. Bouchet. Multimatroids II. Orthogonality, minors and connectivity. The Electronic Journal of Combinatorics, 5:\#R8, 1998.

[2] T. Brylawski. A combinatorial model for series-parallel networks. Transactions of the American Mathematical Society, 154:1-22, 1971.

[3] B. Chaourar and J. G. Oxley. On series-parallel extensions of uniform matroids. European Journal of Combinatorics, 24:877-879, 2003.

[4] Y.B. Choe and D. G. Wagner. Rayleigh matroids. Combinatorics, Probability and Computing, 15(5):765-781, 2006.

[5] C. C. Cocks. Correlated matroids. Combinatorics, Probability and Computing, 17:511-518, 2008.

[6] C. Greene and T. L. Magnanti. Some abstract pivot algorithms. SIAM Journal of Applied Mathematics, 29(3):530-539, 1975.

[7] J.P.S. Kung. Twelve views of matroid theory. In S. Hong, J.H. Kwak, K.H. Kim, and F.W. Roush, editors, Combinatorial and Computational Mathematics: Present and Future, pages 56-96. World Scientific, 2001.

[8] A. P. Mani. Correlation inequalities for Tutte polynomials. PhD thesis, Monash University, 2010.

[9] A. P. Mani. Some inequalities for Whitney-Tutte polynomials. Combinatorics, Probability and Computing, 19(3):425-439, 2010.

[10] S. D. Noble. Private communication, April 2008.

[11] J. G. Oxley. Matroid Theory. Oxford University Press, 1992.

[12] C. Semple and D. J. A. Welsh. Negative correlation in graphs and matroids. Combinatorics, Probability and Computing, 17:423-435, 2008. 
[13] A. D. Sokal. The multivariate Tutte polynomial (alias Potts model) for graphs and matroids. In B.S. Webb, editor, Surveys in Combinatorics, number 327 in London Mathematical Society Lecture Note Series, pages 173-226. Cambridge University Press, 2005.

[14] A. D. Sokal. Private communication, July 2009.

[15] D. G. Wagner. Rank-three matroids are Rayleigh. The Electronic Journal of Combinatorics, 12:\#N8, 2005.

[16] D. G. Wagner. Negatively correlated random variables and Mason's conjecture for independent sets in matroids. Annals of Combinatorics, 12(2):211-239, 2008. 